\title{
Dynamical ejecta of neutron star mergers with nucleonic weak processes - II: kilonova emission
}

\author{
O. Just ${ }^{\oplus, 1,2 \star}$ I. Kullmann, ${ }^{3}$ S. Goriely, ${ }^{3}$ A. Bauswein, ${ }^{1}$ H.-T. Janka ${ }^{\oplus 4}$ and C. E. Collins ${ }^{\oplus 1}$ \\ ${ }^{1}$ GSI Helmholtzzentrum für Schwerionenforschung, Planckstrasse 1, D-64291 Darmstadt, Germany \\ ${ }^{2}$ Astrophysical big bang Laboratory, RIKEN Cluster for Pioneering Research, 2-1 Hirosawa, Wako, Saitama 351-0198, Japan \\ ${ }^{3}$ Institut d'Astronomie et d'Astrophysique, CP-226, Université Libre de Bruxelles, B-1050 Brussels, Belgium \\ ${ }^{4}$ Max-Planck-Institut für Astrophysik, Postfach 1317, D-85741 Garching, Germany
}

Accepted 2021 November 9. Received 2021 November 8; in original form 2021 September 29

\begin{abstract}
The majority of existing results for the kilonova (or macronova) emission from material ejected during a neutron-star (NS) merger is based on (quasi-) one-zone models or manually constructed toy-model ejecta configurations. In this study, we present a kilonova analysis of the material ejected during the first $\sim 10 \mathrm{~ms}$ of a NS merger, called dynamical ejecta, using directly the outflow trajectories from general relativistic smoothed-particle hydrodynamics simulations, including a sophisticated neutrino treatment and the corresponding nucleosynthesis results, which have been presented in Part I of this study. We employ a multidimensional two-moment radiation transport scheme with approximate M1 closure to evolve the photon field and use a heuristic prescription for the opacities found by calibration with atomic-physics-based reference results. We find that the photosphere is generically ellipsoidal but augmented with small-scale structure and produces emission that is about 1.5-3 times stronger towards the pole than the equator. The kilonova typically peaks after $0.7-1.5 \mathrm{~d}$ in the near-infrared frequency regime with luminosities between 3-7 $\times 10^{40} \mathrm{erg} \mathrm{s}^{-1}$ and at photospheric temperatures of $2.2-2.8 \times 10^{3} \mathrm{~K}$. A softer equation of state or higher binary-mass asymmetry leads to a longer and brighter signal. Significant variations of the light curve are also obtained for models with artificially modified electron fractions, emphasizing the importance of a reliable neutrino-transport modelling. None of the models investigated here, which only consider dynamical ejecta, produces a transient as bright as AT2017gfo. The near-infrared peak of our models is incompatible with the early blue component of AT2017gfo.
\end{abstract}

Key words: gravitational waves-nuclear reactions, nucleosynthesis, abundances-radiative transfer-methods: numericalstars: neutron.

\section{INTRODUCTION}

The recent gravitational-wave observation of a binary neutronstar (NS) merger, GW170817 (e.g. Abbott et al. 2017), together with its optical and near-infrared (IR) electromagnetic counterparts, AT2017gfo (e.g. Kasen et al. 2017; Nicholl et al. 2017; Smartt et al. 2017; Tanaka et al. 2017; Tanvir et al. 2017; Villar et al. 2017), provides long-sought observational support to the idea (Lattimer \& Schramm 1976; Eichler et al. 1989) that a substantial fraction of material expelled during NS mergers undergoes the rapid neutroncapture (or r-) process (see e.g. Frebel \& Beers 2018; Kajino et al. 2019; Arnould \& Goriely 2020; Cowan et al. 2021, for recent reviews), which is responsible for the nucleosynthesis of about half of the trans-iron elements in the Universe. Major evidence for this interpretation, hence for the interpretation of AT2017gfo as a socalled kilo- or macronova (e.g. Li \& Paczyński 1998; Kulkarni 2005; Metzger et al. 2010; Metzger 2019), comes from the fact that the light curve of AT2017gfo declined at early times, $t$, roughly as $t^{-1.3}$ characteristic of the energy release rate connected to the consecutive radioactive decay of newly forged r-process elements

^E-mail: o.just@gsi.de
(Metzger et al. 2010; Roberts et al. 2011; Goriely, Bauswein \& Janka 2011). Additional support is provided by spectroscopic features of AT2017gfo, which point to the existence of strontium (Watson et al. 2019) and lanthanides in the ejecta (Kasen et al. 2017; Tanaka et al. 2017). Moreover, the appearance of (at least) two different, red and blue, components in AT2017gfo suggests the existence of multiple channels of matter ejection. Yet, no uniform consensus is reached as to what ejecta component was (mainly) responsible for which kilonova component. The most dominant types of ejecta are the dynamical ejecta (e.g. Ruffert, Janka \& Schaefer 1996; Rosswog et al. 1999; Goriely et al. 2011; Korobkin et al. 2012; Bauswein, Goriely \& Janka 2013; Sekiguchi et al. 2015; Palenzuela et al. 2015; Foucart et al. 2016; Radice et al. 2018b), neutrino-driven winds (e.g. Metzger \& Fernández 2014; Perego et al. 2014; Just et al. 2015a; Fujibayashi et al. 2018; Foucart et al. 2020), and ejecta driven by turbulent viscosity (e.g. Fernández \& Metzger 2013; Just, Obergaulinger \& Janka 2015b; Siegel \& Metzger 2017; Fujibayashi et al. 2018; Miller et al. 2019).

The light curve of a kilonova delivers unique information about the outflow mass, velocity, and composition to the observer. These properties are of paramount importance for, among others, chemical evolution models that attempt to unravel the dominant sites of heavyelement nucleosynthesis (e.g. Shen et al. 2015; Vangioni et al. 2016; 
Côté et al. 2018; van de Voort et al. 2020), in order to understand the evolution and lifetime of the NS remnant and the nuclear equation of state (EoS; e.g. Bauswein et al. 2013; Hotokezaka et al. 2013; Lippuner et al. 2017; Bauswein et al. 2017; Margalit \& Metzger 2017; Radice et al. 2018a; Rezzolla, Most \& Weih 2018; Coughlin et al. 2018; Shibata et al. 2019), or to constrain the processes that launch and collimate ultrarelativistic jets of short gamma-ray bursts (e.g. Just et al. 2016; Ito et al. 2021; Hamidani, Kiuchi \& Ioka 2020; Gottlieb \& Nakar 2021). However, deciphering an observed kilonova light curve remains an ambitious endeavor because of the complexity of involved physics ingredients. Profound theoretical understanding is required of the neutrino-magneto-hydrodynamical matter ejection processes that shape the ejecta structure and set the neutron density (cf. aforementioned references for different ejecta components), of nuclear reaction networks that determine the elemental abundances and the radioactive heating rates powering the emission (e.g. Goriely et al. 2013; Mumpower et al. 2015; Lippuner \& Roberts 2017; Barnes et al. 2021), and of atomic physics and radiative processes that define the opacities and observer-angle dependent emission rates in various frequency bands (e.g. Kasen, Badnell \& Barnes 2013; Fontes et al. 2020; Tanaka et al. 2020).

While tremendous progress has been made in the recent years, each of these aspects still faces considerable challenges and uncertainties. As for the dynamical ejecta, a long-standing uncertainty is connected to their lanthanide fraction and the question whether they would produce rather a red or a blue kilonova, or could even produce both. The lanthanide fraction resulting after the r-process is determined mainly by the electron fraction, or proton-to-baryon ratio, which, in turn, is set during the matter ejection process as a result of the competing emission and absorption processes of electron neutrinos and antineutrinos. Early NS merger simulations, which neglected neutrinos (Bauswein et al. 2013) or included neutrino emission but ignored absorption (Freiburghaus, Rosswog \& Thielemann 1999; Korobkin et al. 2012), found a solar-like abundance pattern for all elements with mass numbers above $A \sim 130-140$ and thus a high lanthanide fraction and correspondingly high opacity. These results implied a kilonova that peaks on rather long time-scales and in red spectral colours (Tanaka \& Hotokezaka 2013; Grossman et al. 2014). Subsequent models using various approximate prescriptions for neutrino absorption revealed that the composition is not as robust as previously thought and may sensitively depend on neutrinotransport effects (Wanajo et al. 2014; Goriely et al. 2015; Martin et al. 2018; Radice et al. 2018b). However, since models including neutrino absorption are still rare and all of them still employ some kind of approximations, and since self-consistent kilonova calculations based on these neutrino-hydrodynamics models are even rarer, the question of the kilonova signature of the dynamical ejecta remains open.

In this study, we take a step towards resolving this question by investigating the kilonova that results from dynamical ejecta modelled by using a state-of-the-art neutrino scheme, namely the Improved Leakage-Equilibration-Absorption Scheme (ILEAS; Ardevol-Pulpillo et al. 2019), which in particular includes neutrinoabsorption effects. The present study follows up on the nucleosynthesis analysis presented in Kullmann et al. (2021; called 'Part I' hereafter) and discusses the kilonova emission for exactly the same models.

Many studies, in fact most of the discovery studies connected to GW170817, are based on a simple but powerful one-zone approximation (e.g. Arnett 1982), which models the ejecta as an expanding homogeneous sphere with a constant, grey (i.e. frequencyindependent) opacity. This approach, or similarly simple considera- tions (e.g. Grossman et al. 2014; Metzger 2019; Hotokezaka \& Nakar 2020), are often employed in a post-processing step to estimate the kilonova emission based on the bulk properties (total mass, average velocity, and average electron fraction) of ejecta obtained in hydrodynamical simulations. Alternatively, schemes are employed that solve the radiative-transfer equations very accurately using state-of-the-art opacity descriptions (Kasen et al. 2013; Tanaka \& Hotokezaka 2013; Wollaeger et al. 2018; Bulla 2019), but which often assume manually constructed toy-model distributions of the ejecta and that are numerically involved and computationally expensive. In this paper, we apply a scheme, based on the M1 approximation of radiative transfer, which in terms of accuracy and complexity, fills the gap between the two aforementioned approaches. Due to its close relationship to hydrodynamics, it can be implemented more readily in existing hydrodynamics codes than schemes solving the full Boltzmann equation. As opposed to most existing studies, we adopt the outflow distributions directly from neutrino-hydrodynamics simulations, and we take the composition and the radioactive heating rate from the consistently post-processed nucleosynthesis tracers.

This paper is organized as follows: In Section 2, we briefly review the hydrodynamic models and corresponding nucleosynthesis results from Part I, describe the employed mapping from hydrodynamic tracers to the velocity space, and outline the governing equations and additional assumptions that enter the kilonova evolution scheme. In Section 3, we present and compare the resulting kilonova light curves. Subsequently, in Section 4, we briefly speculate about the implications of our models to the interpretation of AT2017gfo, point out the advantages of a tracer-based scheme compared to one-zone models, and contrast our results with some published works. Finally, we summarize in Section 5.

\section{METHODOLOGY}

\subsection{Investigated models and adopted quantities}

We study the kilonova emission based on the four neutrinohydrodynamical models of binary NS mergers that have been introduced in Part I. These models have been obtained from smoothedparticle-hydrodynamics (SPH) simulations that were performed in general relativity using the conformal flatness approximation and that employed the recently developed method ILEAS (ArdevolPulpillo et al. 2019) for the treatment of neutrinos. In optically thick regions, ILEAS is consistent with the diffusion law and ensures lepton-number conservation, while in optically thin regions it describes neutrino absorption using an approximation to raytracing. In Part I, the neutrino-hydrodynamics simulations were post-processed by extracting the hydrodynamic properties (density, entropy, and electron fraction), along with the trajectories of all ejected SPH particles and computing the nucleosynthesis yields, along with each of these trajectories using a state-of-the-art nuclear network (see e.g. Lemaître et al. 2021, and references therein). In the four hydrodynamical models, the nuclear EoS is varied between SFHo (Steiner, Hempel \& Fischer 2013) and DD2 (Typel et al. 2010), and the two binary mass configurations $1.35-1.35$ and $1.25-1.45 \mathrm{M}_{\odot}$ are used. We additionally consider three variations of the symmetric DD2 model, which were already introduced in Part I: In model DD2135135-noneu, we ignore all neutrino interactions that take place after the time of the merger, which we define by the time when the general relativistic lapse function reaches a first minimum. This model was denoted as the 'no neutrino' case in Part I. Moreover, to test the impact of uncertainties in the electron fraction, $Y_{e}$, predicted by the neutrino-hydrodynamics simulations, the two models 
Table 1. Global properties of our models as measured in the entire volume (total), measured only in the polar regions defined by the cones of opening angle $\pi / 4$ around the two poles (polar), and measured in the remaining equatorial sub-volume (equat.), namely the ejecta mass, average velocity, average electron fraction, average lanthanide mass fraction, and average initial opacity, as well as the time, bolometric luminosity, surface-averaged photospheric temperature, and surface-averaged photospheric velocity of the resulting kilonova at peak epoch. We define the peak time as the first time when the total luminosity equals the total heating rate, and all other peak quantities are measured at that time. Note that a spherically symmetric mass distribution would correspond to $M_{\mathrm{ej}}^{\text {polar }} / M_{\mathrm{ej}}^{\text {total }}=0.292$.

\begin{tabular}{|c|c|c|c|c|c|c|c|c|c|c|}
\hline Model & Region & $\begin{array}{c}M_{\mathrm{ej}} \\
\left(\mathrm{M}_{\odot}\left(M_{\mathrm{ej}}^{\text {total }}\right)\right)\end{array}$ & $\langle v / c\rangle$ & $\left\langle Y_{e, \rho_{\text {net }}}\right\rangle$ & $\left\langle X_{\mathrm{LA}}\right\rangle$ & $\begin{array}{c}\left\langle\kappa_{\mathrm{LA}}\right\rangle \\
\left(\mathrm{cm}^{2} \mathrm{~g}^{-1}\right)\end{array}$ & $\begin{array}{l}t_{\text {peak }} \\
\text { (d) }\end{array}$ & $\begin{array}{c}L_{\mathrm{peak}} \\
\left(10^{40} \mathrm{erg} \mathrm{s}^{-1}\right)\end{array}$ & $\begin{array}{c}\left\langle T_{\text {ph }}\right\rangle_{\text {peak }} \\
\left(10^{3} \mathrm{~K}\right)\end{array}$ & $\left\langle v_{\text {ph }} / c\right\rangle_{\text {peak }}$ \\
\hline \multirow[t]{3}{*}{ DD2-135135 } & Total & $1.99 \mathrm{e}-3(1)$ & 0.255 & 0.27 & $1.07 \mathrm{e}-1$ & 26.5 & 0.77 & 4.21 & 2.75 & 0.36 \\
\hline & Polar & $3.52 \mathrm{e}-4(0.18)$ & 0.252 & 0.34 & $4.42 \mathrm{e}-2$ & 18.3 & 0.66 & 6.30 & 3.24 & 0.30 \\
\hline & Equat. & $1.62 \mathrm{e}-3(0.82)$ & 0.256 & 0.26 & $1.20 \mathrm{e}-1$ & 28.3 & 0.85 & 3.32 & 2.50 & 0.38 \\
\hline \multirow[t]{3}{*}{ DD2-125145 } & Total & $3.20 \mathrm{e}-3(1)$ & 0.248 & 0.22 & $1.52 \mathrm{e}-1$ & 29.1 & 1.04 & 4.12 & 2.39 & 0.34 \\
\hline & Polar & $5.01 \mathrm{e}-4(0.16)$ & 0.255 & 0.29 & $6.60 \mathrm{e}-2$ & 22.3 & 0.88 & 5.98 & 2.98 & 0.28 \\
\hline & Equat. & $2.66 \mathrm{e}-3(0.84)$ & 0.247 & 0.21 & $1.68 \mathrm{e}-1$ & 30.4 & 1.15 & 3.35 & 2.19 & 0.35 \\
\hline \multirow[t]{3}{*}{ SFHo-135135 } & Total & $3.29 \mathrm{e}-3(1)$ & 0.302 & 0.26 & $1.15 \mathrm{e}-1$ & 27.2 & 0.91 & 5.34 & 2.49 & 0.43 \\
\hline & Polar & $5.49 \mathrm{e}-4(0.17)$ & 0.241 & 0.30 & $8.15 \mathrm{e}-2$ & 24.6 & 0.78 & 7.53 & 2.96 & 0.33 \\
\hline & Equat. & $2.70 \mathrm{e}-3(0.83)$ & 0.314 & 0.25 & $1.22 \mathrm{e}-1$ & 27.8 & 0.99 & 4.24 & 2.26 & 0.46 \\
\hline \multirow[t]{3}{*}{ SFHo-125145 } & Total & $8.67 \mathrm{e}-3(1)$ & 0.241 & 0.24 & $1.14 \mathrm{e}-1$ & 27.4 & 1.49 & 7.51 & 2.20 & 0.37 \\
\hline & Polar & $1.77 \mathrm{e}-3(0.20)$ & 0.225 & 0.28 & $6.62 \mathrm{e}-2$ & 23.2 & 1.33 & 9.64 & 2.52 & 0.33 \\
\hline & Equat. & $6.75 \mathrm{e}-3(0.80)$ & 0.244 & 0.23 & $1.27 \mathrm{e}-1$ & 28.5 & 1.57 & 6.72 & 2.09 & 0.38 \\
\hline \multirow[t]{3}{*}{ DD2-135135-noneu } & Total & $1.99 \mathrm{e}-3(1)$ & 0.255 & 0.13 & $2.46 \mathrm{e}-1$ & 32.3 & 0.86 & 2.79 & 2.35 & 0.35 \\
\hline & Polar & $3.53 \mathrm{e}-4(0.18)$ & 0.252 & 0.17 & $2.22 \mathrm{e}-1$ & 32.0 & 0.73 & 3.80 & 2.67 & 0.30 \\
\hline & Equat. & $1.62 \mathrm{e}-3(0.82)$ & 0.256 & 0.12 & $2.51 \mathrm{e}-1$ & 32.4 & 0.96 & 2.30 & 2.20 & 0.36 \\
\hline \multirow[t]{3}{*}{ DD2-135135-Ye-01 } & Total & $1.99 \mathrm{e}-3(1)$ & 0.255 & 0.17 & $2.02 \mathrm{e}-1$ & 30.7 & 0.85 & 3.18 & 2.47 & 0.35 \\
\hline & Polar & $3.53 \mathrm{e}-4(0.18)$ & 0.252 & 0.24 & $1.37 \mathrm{e}-1$ & 27.6 & 0.70 & 4.69 & 3.04 & 0.29 \\
\hline & Equat. & $1.62 \mathrm{e}-3(0.82)$ & 0.256 & 0.16 & $2.16 \mathrm{e}-1$ & 31.4 & 9.48 & 2.55 & 2.25 & 0.36 \\
\hline \multirow[t]{3}{*}{ DD2-135135-Ye+01 } & Total & $1.99 \mathrm{e}-3(1)$ & 0.255 & 0.37 & $2.31 \mathrm{e}-2$ & 12.2 & 0.63 & 5.09 & 3.14 & 0.38 \\
\hline & Polar & $3.53 \mathrm{e}-4(0.18)$ & 0.252 & 0.44 & $1.04 \mathrm{e}-2$ & 9.42 & 0.53 & 7.36 & 3.23 & 0.34 \\
\hline & Equat. & $1.62 \mathrm{e}-3(0.82)$ & 0.256 & 0.36 & $2.59 \mathrm{e}-2$ & 12.9 & 0.70 & 3.88 & 2.96 & 0.39 \\
\hline
\end{tabular}

DD2-135135-Ye-01 and DD2-135135-Ye+01 consider the cases where along all trajectories $Y_{e}$ was artificially decreased or increased by 0.1 , respectively. Table 1 summarizes the ejecta masses, velocities, average electron fractions, and average lanthanide mass fractions for all models. See Part I for more details regarding the underlying neutrino-hydrodynamics simulations, method of ejecta extraction, employed nuclear network, and the nucleosynthesis results of all models.

\subsection{Data mapping}

Ideally, we would conduct the hydrodynamical simulations of the ejecta until they reach homology, i.e. until the thermal pressure becomes dynamically irrelevant, mixing ceases, and the velocities of Lagrangian fluid elements freeze out. For this study, however, we avoid the significant computational efforts required to follow the long-term expansion and simply assume that homology is already reached at the times $t_{\text {hyd }}$ when the hydrodynamical simulations have been stopped, where $t_{\text {hyd }} \approx 10-20 \mathrm{~ms}$ after the NSs fall into each other. While this assumption is likely to be justified for the high-velocity component of the dynamical ejecta, it may be less appropriate for the slower outflow particles. At $t=t_{\text {hyd }}$ roughly 10-20 per cent of the total ejecta energy still resides in thermal energy, suggesting that the average velocities could still increase by about $5-10$ per cent. We note that our approach of fixing the velocities early after the merger also implies that we cannot describe fallback of gravitationally bound material (e.g. Fernández et al. 2015; Ishizaki et al. 2021) or the dynamical impact of radioactive r-process heating (e.g. Rosswog et al. 2014; Klion et al. 2021a).

Our method of computing the kilonova adopts as input the hydrodynamic properties and nucleosynthesis data of exactly the same outflow trajectories as discussed in Part I. Homologous expansion means that the radial coordinate, $r$, can be replaced by the radial velocity, $v$, because $v(r, t)=r / t$ holds at any given time $t$. Since our kilonova solver operates on an axisymmetric finite-volume grid, we first need to map all required data from particle trajectories, along which the nucleosynthesis calculations have been performed, on to a $2 \mathrm{D}$ spherical polar grid that is spanned in velocity space by the normalized radial coordinate

$$
x \equiv \frac{r}{c t}
$$

(with the speed of light $c$ ) and polar angle, $\theta$. The coordinates of each trajectory particle in this velocity space are given by its radial velocity and the polar angle ${ }^{1}$ along which it is ejected, both measured at $t=t_{\text {hyd }}$. For our calculations, we ignore ejecta in the Southern hemisphere and assume equatorial symmetry, which is found to be rather well fulfilled as shown in Part I. Having identified the position of each ejecta particle in axisymmetric velocity space, we can now interpolate the quantities needed for the upcoming kilonova evolution to all cells of the 2D velocity grid. We map altogether five quantities, namely the mass density, $\rho$, the electron fraction at the onset of network calculations, $Y_{e}\left(\rho_{\text {nuc }}\right)$, the specific heating rate, $Q_{\text {heat }}(t)$ (defined in Section 2.4), and the lanthanide mass fraction, ${ }^{2} X_{\mathrm{LA}}(t)$, as well as the average baryon mass, $\left\langle A_{\text {nuc }}\right\rangle(t)$. Note that $Y_{e}\left(\rho_{\text {nuc }}\right)$ is extracted merely for diagnostic purposes (e.g. Fig. 2) but otherwise

\footnotetext{
${ }^{1}$ The centre and orientation of the axisymmetric spherical polar coordinate system is uniquely defined by the vector of the total orbital angular momentum of the stellar binary and the centre of mass. As usual, the polar angle, $\theta$, is the angle between the coordinate vector and the north pole.

${ }^{2}$ As in Part I, we subsume both lanthanides and actinides under what we call lanthanide fraction here.
} 
not needed by our kilonova scheme, because the dependence of the kilonova on the electron fraction is already included in the quantities $Q_{\text {heat }}, X_{\mathrm{LA}}$, and $\left\langle A_{\text {nuc }}\right\rangle$ provided by the nucleosynthesis calculations. While $\rho$ and $Y_{e}\left(\rho_{\text {nuc }}\right)$ only need to be mapped once ${ }^{3}$ the remaining quantities are time dependent and therefore need to be mapped for a sufficiently large number $(\sim 1000$ in our case $)$ of discrete times, between which linear interpolation will be used in the upcoming evolution scheme.

For the mapping, we adopt interpolation methods that are well known from SPH schemes (see e.g. Price \& Monaghan 2007). We stress, however, that our mapping procedure is completely independent of the SPH code that was used to perform the hydrodynamical simulations. Thus, the kilonova scheme presented here can equally well be applied in cases where the outflow trajectories have been extracted from grid-based simulations. For a set of $N$ outflow particles with masses $m_{j}$ and position vectors $\mathbf{x}_{j}$ (where $\mathbf{x} \equiv x \mathbf{e}^{r}(\theta)$ with radial unit vector $\mathbf{e}^{r}$ and $j=1, \ldots, N$ ), we obtain the density at an arbitrary position $\mathbf{x}$ using

$\rho(\mathbf{x})=\frac{1}{2 \pi \tilde{R}} \sum_{j} m_{j} W_{2 \mathrm{D}}\left(\mathbf{x}-\mathbf{x}_{j}, h_{j}\right)$,

while all other quantities, represented by $A$, are interpolated as

$A(\mathbf{x})=\frac{\sum_{j}^{N} \frac{m_{j}}{\rho_{2 \mathrm{D}, j}} A_{j} W_{2 \mathrm{D}}\left(\mathbf{x}-\mathbf{x}_{j}, h_{j}\right)}{\sum_{j}^{N} \frac{m_{j}}{\rho_{2 \mathrm{D}, j}} W_{2 \mathrm{D}}\left(\mathbf{x}-\mathbf{x}_{j}, h_{j}\right)}$,

from the corresponding values at the particle positions, $A_{j}$. In the above equations, $W_{2 \mathrm{D}}$ is the $2 \mathrm{D}$ cubic spline kernel (see e.g. Monaghan 1992), $h_{j}$ is the smoothing length,

$\rho_{2 \mathrm{D}, j}=\sum_{i}^{N} m_{i} W_{2 \mathrm{D}}\left(\mathbf{x}_{j}-\mathbf{x}_{i}, h_{i}\right)$

is the particle representation of the $2 \mathrm{D}$ density, $\rho_{2 \mathrm{D}} \equiv 2 \pi R \rho$ (e.g. García-Senz et al. 2009), $R$ is the cylindrical radius in normalized velocity space, and $\tilde{R}=\max \{R, h / 2\}$ with $h$ interpolated from $h_{j}$ using equation (3). As commonly done in SPH schemes, we fix the smoothing length, $h_{j}$, by the condition that any sphere of radius $h_{j}$ around particle $j$ should contain roughly the same number of particles ( $\sim 50$ in our case; see e.g. Price \& Monaghan 2007, for explicit equations). In order to reduce numerical artefacts close to the polar axis, namely at cylindrical radii $R \ll h$ that are unresolved by the particle data, we limit the conversion factor $(2 \pi R)^{-1}$ between $\rho_{2 \mathrm{D}}$ and $\rho$ from below by replacing $R$ with $\tilde{R}$ in equation (2).

\subsection{Evolution equations}

After mapping the particle data on to the grid, we compute the kilonova light curves in a simplified fashion using a truncated two-moment scheme with analytic closure (or often called M1scheme; e.g. Minerbo 1978; Levermore 1984; Audit et al. 2002; Just et al. 2015b). Instead of evolving the specific intensity $I_{v}(\mathbf{x}, \mathbf{n})$ as function of the photon frequency $v$ and photon momentum unit vector $\mathbf{n}$, as is done in full-fledged radiative transfer schemes (e.g. Kasen, Thomas \& Nugent 2006; Tanaka \& Hotokezaka 2013), our approximate scheme evolves the 0 th and 1 st angular moments of $I_{v}$ as measured in the comoving (or fluid) frame, namely the

${ }^{3}$ For homologous expansion, the density at any given time can be obtained using $\rho \propto t^{-3}$. monochromatic energy density,

$E_{\nu}(\mathbf{x}) \equiv \frac{1}{c} \int I_{\nu} \mathrm{d} \Omega$

and the monochromatic energy-flux density vector,

$F_{v}^{i}(\mathbf{x}) \equiv \int I_{v} n^{i} \mathrm{~d} \Omega$,

where $\int \mathrm{d} \Omega$ denotes angular integration over the full sphere in photon momentum space. The $\mathcal{O}(v / c)$ evolution equations for these quantities as function of the velocity coordinate vector $\mathbf{x}$ can be derived from the ordinary two-moment equations (e.g. Mihalas \& Mihalas 1984; Just et al. 2015b) by making use of the homology condition, $v(r, t)=r / t$, and they are given by (see e.g. Pinto \& Eastman 2000; Rosswog et al. 2018 for an analog derivation of the Oth-moment equation):

$\frac{\mathrm{d} E_{v}}{\mathrm{~d} t}+\frac{1}{c t} \nabla_{\mathbf{x}} \cdot \mathbf{F}_{v}+\frac{4 E_{v}}{t}-\frac{1}{t} \frac{\partial}{\partial v}\left(v E_{v}\right)=c \kappa\left(E_{v}^{\mathrm{eq}}-E_{v}\right)$,

$\frac{\mathrm{d} \mathbf{F}_{v}}{\mathrm{~d} t}+\frac{c}{t} \nabla_{\mathbf{x}} \cdot \mathbf{P}_{v}+\frac{4 \mathbf{F}_{v}}{t}-\frac{1}{t} \frac{\partial}{\partial v}\left(v \mathbf{F}_{v}\right)=-c \kappa \mathbf{F}_{v}$,

where $\kappa$ is the absorption opacity (see Section 2.4 for the computation), $E_{v}^{\mathrm{eq}}$ is the equilibrium (Bose-Einstein) energy density, and $\mathbf{P}_{v} / E_{v}$ is the normalized 2nd moment (Eddington) tensor with the components

$\frac{P_{v}^{i j}}{E_{v}} \equiv \frac{1}{c E_{v}} \int I_{v} n^{i} n^{j} \mathrm{~d} \Omega$

The M1 approximation consists of assuming that the Eddington tensor is given in terms of a local closure relation as a function of $E_{v}$ and $\mathbf{F}_{v}$. We employ the closure relation by Minerbo (1978). In equations (7) the time derivative $\mathrm{d} / \mathrm{d} t$ is taken at constant velocity, $\mathbf{x}$, and the spatial derivatives, $\nabla_{\mathbf{x}}$, are taken with respect to $\mathbf{x}$. The individual terms entering the time derivative in equations (7) describe, from left to right, the propagation of radiation fluxes, losses due to expansion, Doppler-shift, and (emission and absorption) interactions with ions and electrons.

The energy equation of photons (cf. equation 7a), is coupled to the energy equation for the remaining particles - which will collectively be denoted as gas or fluid in this work - via the first law of thermodynamics for Lagrangian fluid elements moving with velocity $v=r / t$,

$\frac{\mathrm{d} e}{\mathrm{~d} t}+\frac{5 e}{t}=\rho Q_{\text {heat }}-\int_{0}^{\infty} c \kappa\left(E_{v}^{\mathrm{eq}}-E_{v}\right) \mathrm{d} \nu$,

where $e$ is the thermal energy density of the gas. Equation (9) takes into account $p \mathrm{~d} V$ expansion work, energy input from radioactive decay of freshly synthesized elements that powers the kilonova (see Section 2.4 for its computation), and exchange of energy due to emission and absorption of radiation. Since by the time $t>t_{\mathrm{KN}}$, where $0.01 \mathrm{~d} \lesssim t_{\mathrm{KN}} \lesssim 100 \mathrm{~d}$ are typical kilonova emission timescales, most electrons are recombined and free neutrons, protons, and positrons have disappeared, the EoS of the gas is dominated by heavy ions and given by

$e=\frac{3 \rho k_{B} T}{2\left\langle A_{\mathrm{nuc}}\right\rangle m_{u}}$,

where $T, k_{B}$, and $m_{u}$ are the fluid temperature, Boltzmann constant, and atomic mass unit, respectively, and the average mass number of ions, $\left\langle A_{\text {nuc }}\right\rangle$, is provided by the nucleosynthesis calculations.

The evolution during the intermediate phase between the merger and the kilonova emission, namely during $t_{\text {hyd }}<t<t_{\mathrm{KN}}$, is less important, because in this adiabatic phase the total (photon plus 
gas) energy quickly converges towards a time-dependent quasiequilibrium that is determined by the balance between adiabatic expansion and radioactive heating. Hence, as long as the time of initialization, $t_{0}$, is chosen to be early enough for the system to reach the quasi-equilibrium well before $t \approx t_{\mathrm{KN}}$, the resulting light curve should be insensitive to the particular choice of initial conditions. Motivated by sensitivity tests using different values of $t_{0}$ and different initial gas temperatures (see Appendix B) we initiate our simulations at $t=100 \mathrm{~s}$ after the merger using as initial conditions a homogeneous temperature of $T=100 \mathrm{~K}$ and negligibly small radiation energies. The duration of our kilonova simulations is constrained by the fact that we assume local thermodynamic equilibrium (LTE), which implies that our scheme is not applicable at late times, typically beyond $t \sim 5-20 \mathrm{~d}$ (depending on the ejecta properties) when non-LTE effects become dominant (e.g. Waxman, Ofek \& Kushnir 2019). We furthermore note that the possibility of a neutron precursor (Metzger et al. 2015), which could be produced through the decay of free neutrons in the fastest $(v / c \gtrsim 0.5)$ layers of the ejecta, is not discussed in this study, mainly because such a signal cannot properly be described by our $\mathcal{O}(v / c)$ scheme.

The numerical methods employed to solve the M1 equations, of which the canonical form can be recovered by rescaling the time coordinate as shown in appendix B of Just et al. (2022), are detailed in Just et al. (2015b). We employ the same code ALCAR that is described in Just et al. (2015b) and was previously used to evolve the M1 equations for neutrino transport.

A few comments are in order regarding the advantages and disadvantages of the M1 scheme. While the M1 scheme has been employed already in a large number of previous applications in the context of photon and neutrino transport (e.g. Cernohorsky \& Bludman 1994; Smit, van den Horn \& Bludman 2000; Pons, Ibáñez \& Miralles 2000; Audit et al. 2002; McKinney et al. 2014; Just et al. 2015b; O’Connor 2015; Kuroda, Takiwaki \& Kotake 2016; Weih et al. 2020) the present scheme is, to our knowledge, the first application to the computation of kilonovae. A compelling advantage of the M1 scheme is its computational efficiency and algorithmic simplicity compared to full-scale radiative transfer schemes that resolve the angular distribution of the photon field. The hyperbolic nature of the M1 equations allows to integrate spatial derivatives explicitly in time and by that avoids inversions of large matrices during each integration step (e.g. Just et al. 2015b), keeping the computational expense comparable to that of hydrodynamic solvers. The accuracy of the scheme is generally expected to be superior to some widely employed, more approximate methods. For instance, the leakage-like model by Grossman et al. (2014) does not solve a conservation equation for the photon energy, and as a consequence it systematically underestimates the luminosities around peak epoch. ${ }^{4}$ In contrast to (quasi-) one-zone approximations that do solve an energy conservation equation for photons (e.g. Arnett 1982; Goriely et al. 2011; Villar et al. 2017; Metzger 2019; Hotokezaka \& Nakar 2020), the M1 formulation does not depend on manually chosen estimates of the diffusion rate, because it self-consistently resolves the spatial propagation of radiation through the ejecta. Moreover, it is able to consistently describe heating due to re-absorption of photons

\footnotetext{
${ }^{4}$ This is because the luminosity estimated in the model by Grossman et al. (2014) is always bound to be lower than or equal to the current global heating rate, which is inconsistent with more detailed calculations where the luminosity typically exceeds the heating rate at times close to the peak. We refer the reader to the appendix of Rosswog et al. (2018) for a comparison of the model by Grossman et al. (2014) with more accurate schemes.
}

transported from one location to another, and it provides a genuinely multidimensional framework that can handle non-radial fluxes.

On the other hand, the main disadvantage of the M1 scheme is its poor ability to describe radiation in the optically thin regime, particularly in the case of geometrically complex radiation sources (e.g. Audit et al. 2002; Just et al. 2015b; Weih et al. 2020). While the underlying Boltzmann equation predicts linear superposition of radiation packets in the optically thin regime, the non-linear closure relation of the M1 scheme causes radiation beams to interact with each other even where collisional interaction rates vanish. A particularly noteworthy consequence of this shortcoming is that radiation emitted in the optically thin phase of evolution, after the photosphere has disappeared, is not isotropic, as it should be, but still retains a certain dependence on the observer angle, $\theta_{\text {obs }}$ (cf. Section 3.2). In view of the approximate handling in M1 of the angular photon distribution function, we consider the obtained dependence of radiation fluxes on specific observer angles to be less reliable than corresponding averages over finite angle intervals. This is why we will restrict most of our discussion to considering luminosities averaged over finite solid angle domains instead of ones measured at specific angles.

\subsection{Heating rate and opacity}

The quantity $Q_{\text {heat }}$ on the right-hand side of equation (9) is computed as

$Q_{\text {heat }} \equiv f_{\text {th }}\left(Q+Q_{\text {neu }}\right)$

and represents the effective heating rate per unit of mass, namely the fraction $f_{\text {th }}$ of the total radioactive energy release rate, $Q+Q_{\text {neu }}$, which is converted to thermal energy on time-scales shorter than the evolution time-scale. We follow the notation of Part I and use $Q$ to denote the release rate without neutrinos, while $Q_{\text {neu }}$ stands for the contribution that is carried away by neutrinos (and not available for thermal heating in any case). We compute the thermalization efficiency, $f_{\text {th }}$, exactly as in Rosswog et al. (2017; who adopted the formalism by Barnes et al. 2016), namely as

$f_{\text {th }}=\frac{Q_{\beta}\left[\tilde{\zeta}_{\gamma} f_{\gamma}+\tilde{\zeta}_{e} f_{e}\right]+Q_{\alpha} f_{\alpha}+Q_{\text {fis }} f_{\text {fis }}}{Q+Q_{\text {neu }}}$,

where $Q_{\beta}, Q_{\alpha}$, and $Q_{\text {fis }}$ are the partial release rates ${ }^{5}$ from $\beta$ decay, $\alpha$-decay, and fission, and the thermalization efficiencies $f_{i}$ ( $i \in\{\gamma, e, \alpha$, fis $\}$ ) for photons, electrons, $\alpha$ particles, and fission products are functions of $\tau_{i} / t$ with the thermalization time-scales $\tau_{i}$; see Rosswog et al. (2017) for the explicit expressions. Since in our notation, $Q_{\beta}$ does not contain the energy release going into neutrinos, the weighting factors $\tilde{\zeta}_{\gamma}=0.69$ and $\tilde{\zeta}_{e}=0.31$ differ from the ones $\left(\zeta_{\gamma}=0.45\right.$ and $\left.\zeta_{e}=0.2\right)$ employed in Rosswog et al. (2017). Note that while the time-scales $\tau_{i}$ are functions only of the total mass and average velocity of the ejecta, $f_{\text {th }}$ can be different along each trajectory because of different relative contributions of $Q_{\beta}, Q_{\alpha}$, and $Q_{\text {fis. }}$. The average thermalization efficiencies for all investigated models as functions of time are displayed in Fig. 1. Almost perfect thermalization $\left(Q_{\text {heat }} \approx Q\right)$ prevails until about $t \sim 0.5-1 \mathrm{~d}$, whereafter $f_{\text {th }}$ declines roughly as $\propto t^{-1}$. Lower ejecta masses or faster expansion velocities accelerate the decay of $f_{\text {th }}$. For completeness, we also plot the fraction $f_{\text {neu }} \equiv Q_{\text {neu }} /\left(Q+Q_{\text {neu }}\right)$ of energy that is carried away by neutrinos, which amounts to about $\approx 50-60 \$$ per cent in our models.

${ }^{5}$ See section 3.2 and fig. 9 in Part I for a discussion of the individual contributions to $Q$. 

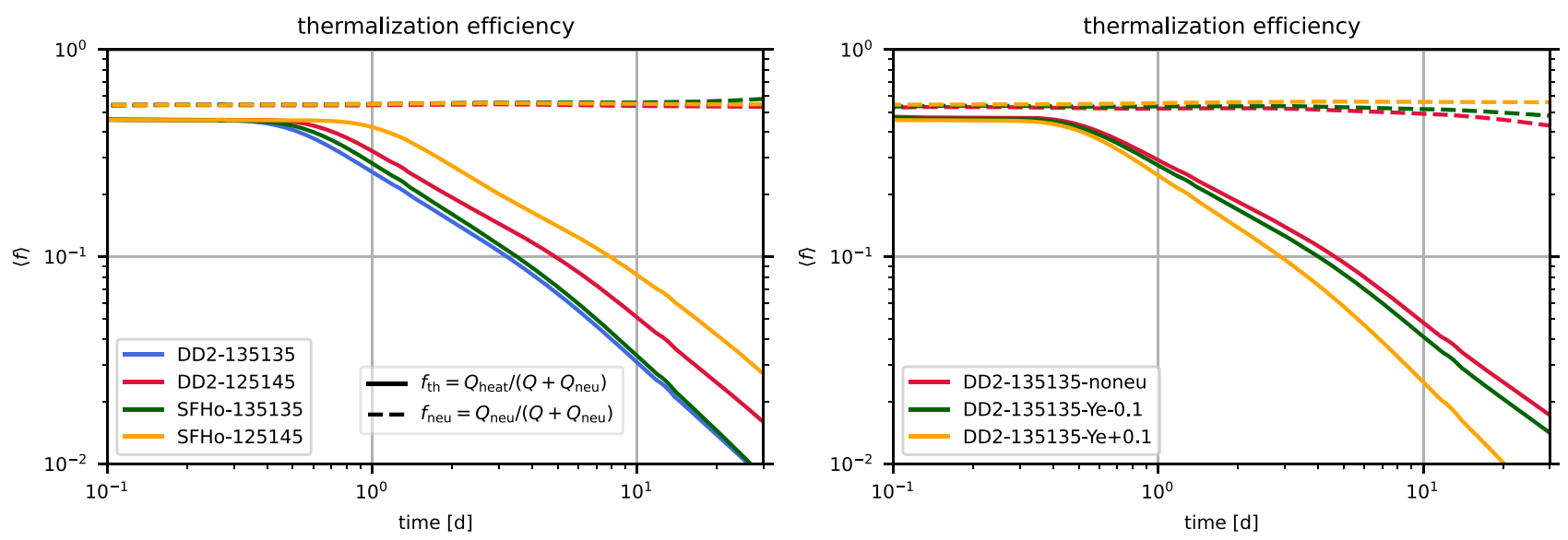

Figure 1. Mass-averaged thermalization efficiencies as function of time for all of our models as computed with equation (12; solid lines), as well as the mass-averaged fraction of the radioactive energy release rate that is carried away by neutrinos (dashed lines).

Finally, we need a reasonable prescription for the opacities, which are dominated mainly by thousands of possible transition lines between energy levels of $f$-shell elements newly created by the $\mathrm{r}$ process. Instead of using a detailed atomic-physics based opacity model (e.g. Kasen et al. 2013; Wollaeger et al. 2018; Tanaka et al. 2020) in our approximate kilonova solver, we express the opacity in a parametric fashion as a function of the lanthanide mass fraction and gas temperature as

$\kappa\left(X_{\mathrm{LA}}, T\right)=\kappa_{\mathrm{LA}} \times \kappa_{T}$

where the $X_{\mathrm{LA}}$-dependent part is

$\kappa_{\mathrm{LA}} \equiv \begin{cases}30 \mathrm{~cm}^{2} \mathrm{~g}^{-1}\left(X_{\mathrm{LA}} / 10^{-1}\right)^{0.1} & , X_{\mathrm{LA}}>10^{-1}, \\ 3 \mathrm{~cm}^{2} \mathrm{~g}^{-1}\left(X_{\mathrm{LA}} / 10^{-3}\right)^{0.5} & , 10^{-3}<X_{\mathrm{LA}}<10^{-1}, \\ 3 \mathrm{~cm}^{2} \mathrm{~g}^{-1}\left(X_{\mathrm{LA}} / 10^{-3}\right)^{0.3} & , 10^{-7}<X_{\mathrm{LA}}<10^{-3}, \\ 0.2 \mathrm{~cm}^{2} \mathrm{~g}^{-1} & , X_{\mathrm{LA}}<10^{-7},\end{cases}$

and the temperature-dependent part is

$\kappa_{T} \equiv \begin{cases}1 & , T>2000 \mathrm{~K} \\ \left(\frac{T}{2000 \mathrm{~K}}\right)^{5} & , T<2000 \mathrm{~K} .\end{cases}$

The above prescription was motivated by fits to bolometric light curves from the atomic-physics based models of Kasen et al. (2017); see Appendix A for comparison plots. Our simplified opacities are not able to reproduce all quantitative features in all models by Kasen et al. (2017), but they produce agreement in the bolometric luminosities typically within a factor of two, and they rather reliably predict the correct frequency regime and epoch of maximum brightness of individual broadband light curves. While our prescription is only a crude approximation to atomic-physics based models, it is more advanced than many previous treatments using (piecewise) constant opacities (e.g. Perego, Radice \& Bernuzzi 2017; Villar et al. 2017; Rosswog et al. 2018; Hotokezaka \& Nakar 2020) in two important respects: First, the functional dependence between $X_{\mathrm{LA}}$ and $\kappa$ is more consistent with sophisticated opacities. In particular, it ensures that variations in the lanthanide fractions always result in variations of the opacity and therefore of the light curve, which is not guaranteed for a piecewise constant prescription. Secondly, the temperature-dependent reduction factor, $\kappa_{T}$, incorporates the effect that electron recombination and the concomitant disappearance of atomic transition lines lead to a decline of the opacities at late times (e.g. Kasen et al. 2013; Tanaka et al. 2020; Zhu et al. 2021).

\subsection{Grid setup}

The hydrodynamical NS-NS merger simulations sample the outflow in each model with $N \sim 800-1400$ trajectories (see Part I). The kilonova evolution is simulated on a velocity grid containing about 400 zones in radial direction, distributed uniformly within $v / c=$ $0.02-1.2,{ }^{6}$ and 60 equidistant zones in $\theta$ direction from 0 to $\pi / 2$. Frequency space is discretized by 50 frequency bins, which are distributed logarithmically between $v=0$ and $2.42 \times 10^{15} \mathrm{~Hz}$. The mid-points of the outermost bins are located at wavelengths of $130 \mathrm{~nm}$ and $289 \mu \mathrm{m}$.

\subsection{Extraction of observer light curves}

In contrast to a complete radiative transfer solver, the approximate two-moment system with analytic closure that we solve, equation (7), does not evolve the angular distribution of photons in momentum space, but only the 0th- and 1st-angular moments thereof (cf. equations 5 and 6). Since the moments are defined in the frame locally comoving with the ejecta the fluxes $F_{v}^{i}$ measured at some velocity coordinate $v_{m}$ above the photosphere are Doppler red-shifted and retarded in time compared to the fluxes measured by a Eulerian observer, who is assumed to be at rest with respect to the centre of mass of the NS binary. Moreover, due to the proximity of $v_{m}$ to spatially extended cloud of ejecta a significant fraction of photons is still moving in lateral directions, i.e. the angular distribution of $F_{v}^{r}$ is not the final one as measured by an observer. We apply the following scheme based on discretizing the radiation field into radiation packets in order to reconstruct the approximate angular distribution of the radiation field and to correct for the frame dependence (inspired by a related treatment in Lucy 2005).

The first step consists of recording the comoving-frame radiation moments, $E_{v}, F_{v}^{r}$, and $F_{v}^{\theta}$ at the time-dependent velocity coordinate

$v_{m}(t)=\max \left\{0.5 c, \min \left\{0.99 c, 1.2\left\langle v_{\mathrm{ph}}\right\rangle\right\}\right\}$,

which is chosen to be 20 per cent larger than the average location of the photosphere [see Section 3.2 and equation (29) for the definition

${ }^{6}$ The reason for locating the outer boundary at $v>c$ is only to prevent numerical artifacts, which may be encountered at early times when radiation crosses the boundary with low values of the flux factor, $\left|\mathbf{F}_{v}\right| /\left(c E_{v}\right)$. 
of the photosphere], but within $c$ and $c / 2$. The lower limit of $0.5 c$ is applied in order to ensure that monitoring is performed outside of regions of significant photon production also after the ejecta become optically thin.

In the next step we reconstruct the specific intensity, $I_{\nu}$, using explicitly the closure prescription of Minerbo (1978). This closure assumes $I_{v}$ to be of the form

$I_{\nu}(\tilde{\mu}) \propto e^{a_{\nu} \tilde{\mu}}$

as function of the cosine

$\tilde{\mu} \equiv \mathbf{n} \cdot \frac{\mathbf{F}_{v}}{\left|\mathbf{F}_{v}\right|}$

of the angle between the momentum vector of photons, $\mathbf{n}$, and the flux-density unit vector, $\mathbf{F}_{v} /\left|\mathbf{F}_{v}\right|$, and where the parameter $a_{v}=L^{-1}\left(\left|\mathbf{F}_{v}\right| /\left(c E_{v}\right)\right)$ with the inverse of the Langevin function, $L^{-1}$. The normalization constant of $I_{v}$ is obtained from the definition of either of the evolved moments (cf. equations 5 or 6). Next, we discretize the momentum space of photons, using as coordinates $\mu \equiv \mathbf{n} \cdot \mathbf{e}^{r} \in[-1,1]$, the cosine of the angle between the photon momentum and the radial direction, and the azimuthal angle $\Phi$ $\in[0,2 \pi)$ between the projection of $\mathbf{n}$ on to the plane normal to $\mathbf{e}^{r}$ and the unit vector in $\theta$ direction, $\mathbf{e}^{\theta}$. The differential energy of a radiation packet emitted at time $t$, velocity $v=v_{m}(t)$, polar angle $\theta$, and frequency $v$ into the direction of $\mathbf{n}(\mu, \Phi)$ is then given by

$E_{\text {pack }}=I_{\nu}\left(v_{m}, \theta, \mu, \phi, t\right) \mu \mathrm{d} \nu \mathrm{d} \mu \mathrm{d} \Phi \times 2 \pi\left(v_{m} t\right)^{2} \sin \theta \mathrm{d} \theta \mathrm{d} t$

and the number of photons carried by that packet is $N_{\text {pack }}=E_{\text {pack }} /(h v)$ (with Planck constant $h$ ). While $N_{\text {pack }}$ is frame independent, the energy, momentum vector, and arrival time of each packet as measured by an observer at rest transform as

$E_{\text {pack }, \mathrm{obs}}=E_{\mathrm{pack}} \Gamma\left(1+\mathbf{n} \cdot \frac{\mathbf{v}}{c}\right)$,

$\mathbf{n}_{\mathrm{obs}}=\frac{E_{\mathrm{pack}}}{E_{\mathrm{pack}, \mathrm{obs}}}\left[\mathbf{n}+\Gamma \frac{\mathbf{v}}{c}\left(1+\frac{\Gamma}{\Gamma+1} \mathbf{n} \cdot \frac{\mathbf{v}}{c}\right)\right]$,

$t_{\mathrm{obs}}=t\left(1-\mathbf{n}_{\mathrm{obs}} \cdot \frac{\mathbf{v}}{c}\right)$,

where $\mathbf{v} \equiv v_{m} \mathbf{e}^{r}$ and $\Gamma \equiv\left(1-v_{m}^{2} / c^{2}\right)^{-1 / 2}$. Since the time elapsed in the $v=0$ frame is the same as the observer time, we will from now on drop the subscript 'obs' from the observer time coordinate and, for the sake of a compact presentation, use $t$ to denote both ejecta properties and the observer signal.

The differential luminosity, $\mathrm{d} L_{v}$, at time $t_{\text {obs }}$, observer angle $\theta_{\text {obs }}$, and frequency $v$ is then given by the sum of energy packets $E_{\text {pack, obs }}$ arriving between $t_{\mathrm{obs}}$ and $t_{\mathrm{obs}}+\mathrm{d} t$ into the solid-angle interval $\mathrm{d} \Omega=$ $2 \pi \sin \theta_{\text {obs }} \mathrm{d} \theta_{\text {obs }}$ and with mean energy $E_{\text {pack, obs }} / N_{\text {pack }}$ between $h v$ and $h v+h \mathrm{~d} v$, divided by $\mathrm{d} t$. The total luminosity going into a given solid angle is then just the sum of $\mathrm{d} L_{v}$ over the appropriate angle interval. We compute the isotropic-equivalent luminosity into a given observer angle as

$L_{\text {iso }, v}\left(\theta_{\text {obs }}\right)=4 \pi \frac{\mathrm{d} L_{v}}{\mathrm{~d} \Omega}$

and the flux density measured by an observer at distance $d$ as

$F_{v, \mathrm{obs}}\left(\theta_{\mathrm{obs}}\right)=\frac{L_{\mathrm{iso}, v}\left(\theta_{\mathrm{obs}}\right)}{4 \pi d^{2}}$.

Corresponding bolometric quantities, for which we will use the same symbol but without the subscript $v$, are obtained by integration over frequency. Absolute $\mathrm{AB}$ magnitudes are computed from the above fluxes as ${ }^{7}$

$M_{\mathrm{AB}} \equiv-2.5 \times \log _{10}\left\{F_{v, \mathrm{obs}}\left[\operatorname{erg~s}^{-1} \mathrm{~cm}^{-2} \mathrm{~Hz}^{-1}\right]\right\}-48.6$,

while in this study we restrict ourselves to the three bands $g$, $z$, and $H$. The $g$ band represents green optical frequencies, the $z$ band the blue end of the near-IR domain, and the $H$ band the red end of the near-IR domain. For computing the magnitudes in these bands, we adopt the fluxes at the mid-point wavelength of each band, namely at 514,902 , and $1630 \mathrm{~nm}$, respectively, i.e. we do not apply a filter function.

Furthermore, we extract, for each observer angle, $\theta_{\mathrm{obs}}$, and time, $t, \mathrm{a}$ temperature, $T_{\mathrm{obs}}$, and velocity, $v_{\mathrm{obs}}$, by fitting the observed isotropicequivalent luminosities, $L_{\mathrm{iso}, v}$, to a blackbody luminosity, $L_{\mathrm{iso}, v}^{\mathrm{BB}}$, which would result if all photons were emitted with a blackbody spectrum of temperature $T_{\mathrm{obs}}$ from a spherical surface of radius $v_{\mathrm{obs}} t$, where

$L_{\text {iso }, v}^{\mathrm{BB}}\left(T_{\mathrm{obs}}, v_{\mathrm{obs}}\right) \equiv \frac{8 \pi^{2} h\left(v_{\mathrm{obs}} t\right)^{2}}{c^{2}} \frac{v^{3}}{\exp \left\{h v /\left(k_{\mathrm{B}} T_{\mathrm{obs}}\right)\right\}-1}$

with Boltzmann constant $k_{\mathrm{B}}$. The quantities $T_{\text {obs }}$ and $v_{\text {obs }}$ estimate the temperature and velocity of the photosphere based on observable fluxes only. In Sections. 3.1 and 3.2, we will additionally introduce the actual temperature and velocity of the photosphere, $T_{\mathrm{ph}}$ and $v_{\mathrm{ph}}$. We will provide both types of quantities for each model in order to illustrate the connection between properties of the expanding ejecta and the observable signal.

\section{RESULTS}

In the following, we consider the spatial structure of the ejecta and the photosphere in Section 3.1, then we examine the light curves as functions of time and observer angle for a fiducial model, and subsequently we investigate the model dependence of the results.

We summarize global properties of the ejecta and the corresponding kilonova in Table 1. We stress that throughout this paper our definition of the kilonova peak is not strictly mathematical, i.e. we do not identify the peak as the point where the brightness reaches a global maximum. Maximum brightness can appear already very early, in which case it is produced only by a small amount of mass located at the outer edge of the ejecta (see e.g. Banerjee et al. 2020, for a light-curve comparison between different choices of mass distributions in the outermost shells). Since we are not interested in the emission coming from the fastest ejecta shells, but rather in that from the bulk ejecta, we measure the peak as the time when the bolometric luminosity, $L$, first starts to exceed the volume integral of the instantaneous heating rate,

$q \equiv \int_{V} \rho Q_{\text {heat }} \mathrm{d} V$

which signals the onset of the optically thin phase. ${ }^{8}$

\footnotetext{
${ }^{7}$ By convention, absolute magnitudes assume the flux $F_{v, \text { obs }}$ in equation (23) to be measured at a distance of $10 \mathrm{pc}$.

${ }^{8}$ The crossing of $q$ and $L$ is a generic feature of radioactively powered transients (e.g. Arnett 1982; Kasen \& Barnes 2019; Hotokezaka \& Nakar 2020) and a result of the fact that radioactive heating effectively charges the thermal energy content of the ejecta in the late optically thick phase. The subsequent rapid release (called 'diffusion wave'; see e.g. Waxman et al. 2018) of this surplus of energy commencing once optically thin conditions are reached creates luminosities in excess of the instantaneous heating rates.
} 

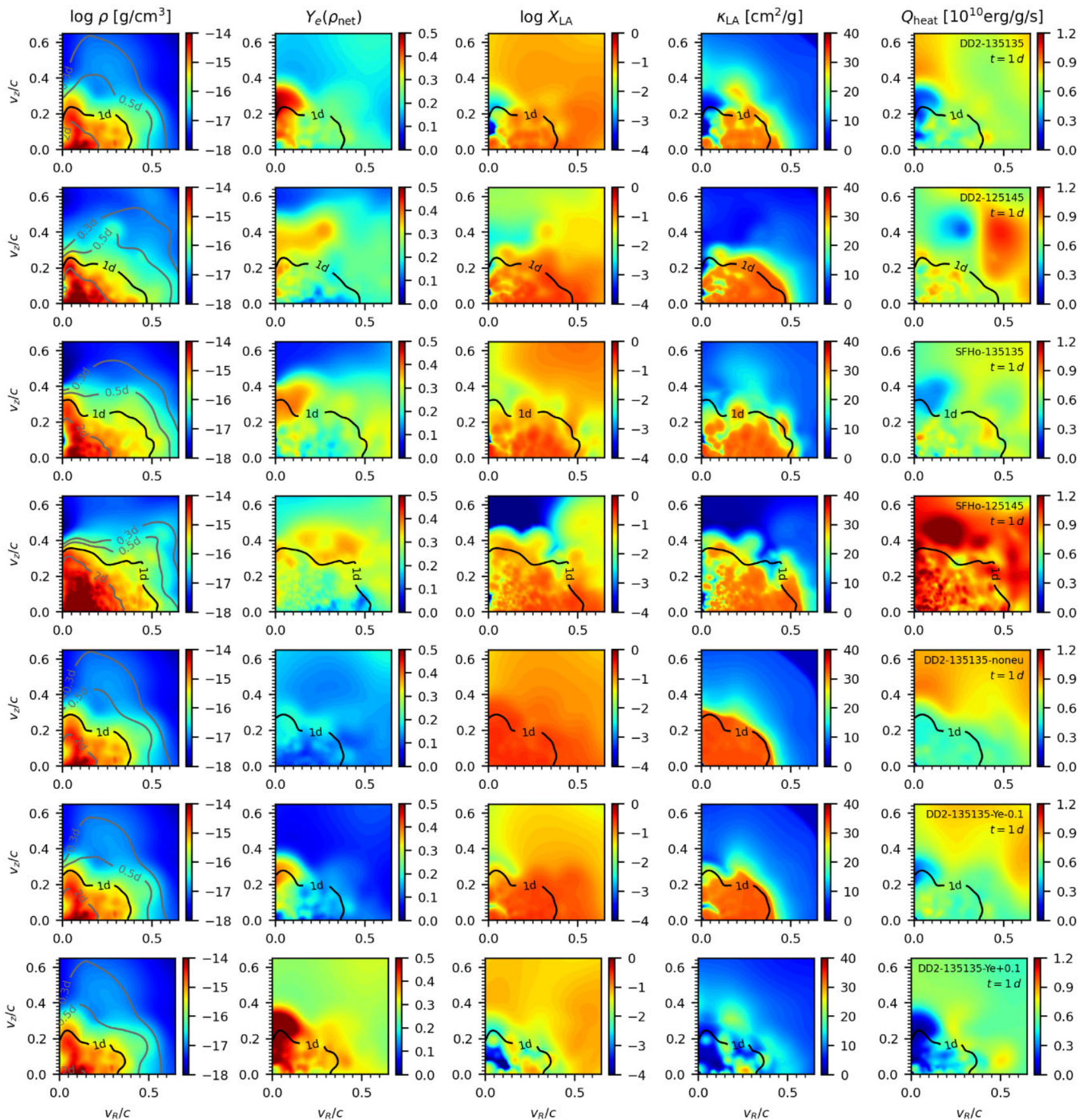

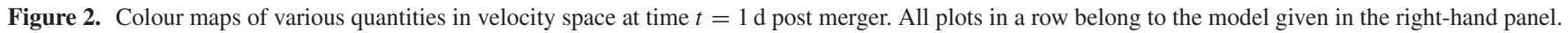

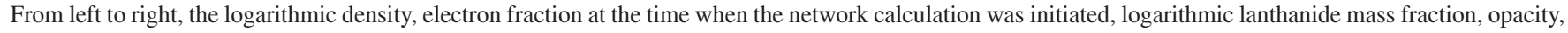

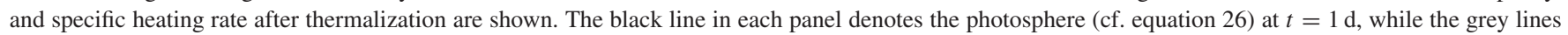
in the left-hand panels additionally show the photospheres at $t=0.3,0.5$, and $2 \mathrm{~d}$ going from high-to-low velocities.

In Table 1, as well as in the following discussions, we often distinguish between polar and equatorial properties. If not explicitly stated otherwise, 'equatorial' always refers to the region within angles of $-\pi / 4$ and $+\pi / 4$ around the equator, while 'polar' refers to the remaining volume of the sphere. ${ }^{9}$

${ }^{9}$ We caution the reader that the two characteristic regions used here are different from the three regions (polar, middle, equatorial) used in Part I.

\subsection{Ejecta structure}

Before considering the light curves, we first inspect the spatial distribution of the ejecta. To that end, we provide in Fig. 2 a tableau of contour plots showing for all models the density, electron fraction, lanthanide fraction, opacity, and heating rate in the polar plane at a time of $t=1 \mathrm{~d}$ post merger. Moreover, Fig. 3 provides for all models the angle-integrated mass distributions as function of the velocity coordinate. 

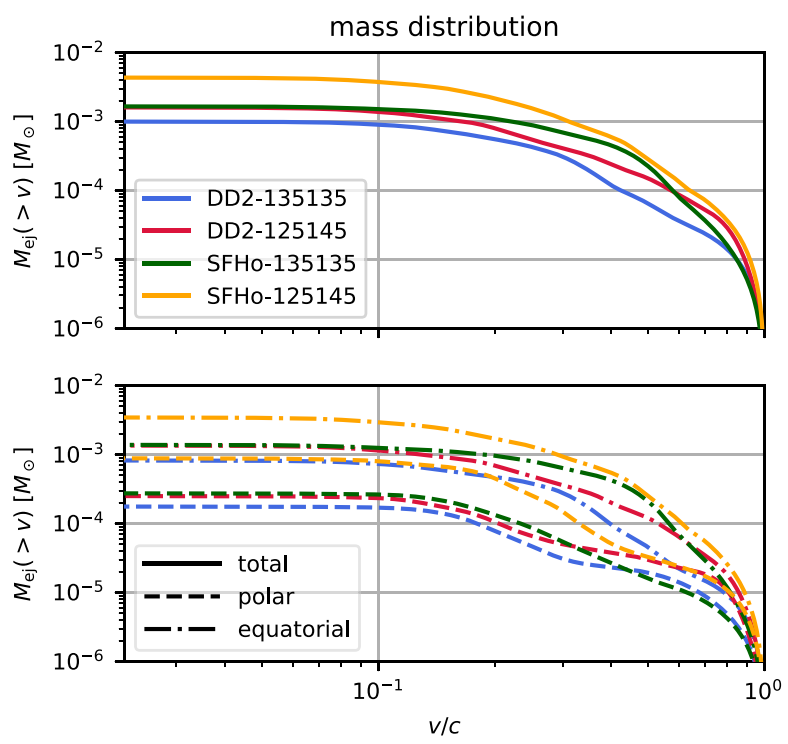

Figure 3. Mass distribution of all investigated models showing for a given velocity $v$ the mass of all ejected material expanding faster than $v$. In the top panel, the angle integration is performed over the entire sphere while in the bottom panel $M_{\mathrm{ej}}(>v)$ is shown separately for polar $(0<\theta<\pi / 4$ and $3 \pi / 4$ $<\theta<\pi)$ and equatorial $(\pi / 4<\theta<3 \pi / 4)$ regions.

As already discussed in Part I, and in qualitative agreement with results by other groups (Sekiguchi et al. 2015; Foucart et al. 2016; Radice et al. 2018b), all models show, at least for velocities $v / c \lesssim 0.5$, a characteristic pole-to-equator asymmetry in the electron fraction, $Y_{e}$, which increases from $Y_{e} \approx 0.15-0.2$ near the equator to about 0.3-0.35 near the poles (cf. panels in second row from left in Fig. 2). High values of $Y_{e}$ beyond $\sim 0.25$ are mainly a result of neutrino absorption, which tends to dominate neutrino emission near the poles and drives $Y_{e}$ towards $\sim 0.5$ (e.g. Goriely 2015; see also Part I). As a result of the reduced neutron-richness, the mass-averaged lanthanide fraction, $\left\langle X_{\mathrm{LA}}\right\rangle$, in the polar cones is about a factor of two smaller than in the equatorial region (cf. Table 1). As a consequence, the mass-averaged opacities, $\left\langle\kappa_{\mathrm{LA}}\right\rangle$, in the corresponding regions differ by about $15-30$ per cent. Besides the composition asymmetry, polar ejecta also exhibit a slightly reduced mass loading compared to equatorial ejecta (cf. bottom panel of Fig. 3). The mass ratio $M_{\mathrm{ej}}^{\text {polar }} / M_{\mathrm{ej}}^{\text {total }}$ of polar to total ejecta is in all models about a factor of $\sim 2$ smaller than the value of 0.292 that would result for an isotropic ejecta distribution (cf. Table 1 and see Part I).

The black and grey lines in Fig. 2 denote the photosphere, which we define as the surface $r_{\mathrm{ph}}(\theta)$ where the radial optical depth, $\tau$, equals unity, i.e.

$\tau\left(r_{\mathrm{ph}}(\theta), \theta\right)=\int_{r_{\mathrm{ph}}}^{\infty} \kappa \rho \mathrm{d} r=1$.

Around times of $t \sim 1 \mathrm{~d}$, i.e. close to the epoch of peak emission, the photospheres of all models resemble, to first order, an oblate ellipsoid with semimajor axial ratio of about 1.2-2. However, the shape of the photosphere varies with time and becomes more spherical or even prolate for some models at very early or late times. Moreover, apart from the global geometry, the ejecta also exhibit a significant degree of clumpiness and small-scale structure, i.e. structures on scales of $\Delta v \sim 0.1 c$ and less. Such variations can be a result of the case dependent merger dynamics, e.g. of mass shedding during the plunge, or oscillations of the merger remnant (e.g. Bauswein et al. 2013), or of other processes such as spiral waves (e.g. Nedora et al. 2019). As a consequence, the ejecta of all investigated models develop a photosphere with wiggles and irregular shapes and a complex time dependence (see left-hand panel of Fig. 2 for photospheres at different times), clearly different from the idealized geometries that were assumed in previous studies (e.g. Kawaguchi, Shibata \& Tanaka 2018; Darbha \& Kasen 2020; Korobkin et al. 2021). The degree of clumpiness seen in our models may be exaggerated by our particlebased interpolation method and the fact that the hydrodynamics are not followed all the way until self-similar expansion, in particular, because r-process heating on longer time-scales may lead to smoother density profiles (Rosswog et al. 2014). On the other hand, our models may also underestimate the level of anisotropy to some extent, because they are azimuthally averaged, do not include magnetic fields (e.g. Ciolfi \& Kalinani 2020), or possibly under-resolve the smallscale structure due to a limited numerical resolution in the ejecta. It is well conceivable that irregular structures in the ejecta have a non-negligible bearing on the kilonova emission, e.g. by enhancing the emission anisotropy. However, since our approximate kilonova treatment is not well suited for reliably tracking the radiative transfer effects of local (in contrast to global) asymmetries in the photospheric region, we will not discuss the ramifications of the ejecta clumpiness for the kilonova light curve in this study, but leave such investigations to future studies based on more accurate radiative transfer solvers.

\subsection{Light curves of fiducial model}

We now discuss the light curve starting with the fiducial model DD2-135135. In Fig. 4, we plot, as functions of time, the angleaveraged bolometric luminosity, the total and mass-averaged specific heating rates, $q$ and $q / M_{\mathrm{ej}}$, respectively, the surface-averaged absolute magnitudes in $g, z$, and $H$ bands, the mass-averaged opacity,

$\langle\kappa\rangle=\frac{\int_{V} \rho \kappa \mathrm{d} V}{M_{\mathrm{ej}}}$,

as well as the surface-averaged temperature and velocity at the photosphere,

$\left\langle T_{\mathrm{ph}}\right\rangle=\frac{1}{2} \int_{0}^{\pi} T\left(r_{\mathrm{ph}}, \theta\right) \sin \theta \mathrm{d} \theta$,

and

$\left\langle v_{\mathrm{ph}}\right\rangle=\frac{1}{2} \int_{0}^{\pi} \frac{r_{\mathrm{ph}}}{t} \sin \theta \mathrm{d} \theta$,

respectively, together with the corresponding surface-averaged estimates $\left\langle T_{\mathrm{obs}}\right\rangle$ and $\left\langle v_{\mathrm{obs}}\right\rangle$ of the photospheric conditions based on observable fluxes.

The bulk of the ejecta starts to become optically thin at about $t \approx$ $0.8 \mathrm{~d}$, which is the time when the luminosity exceeds the heating rate. By the time of $t \approx 3 \mathrm{~d}$, the optical depth has dropped below unity, i.e. the photosphere has disappeared, implying that re-absorption of thermal photons has become irrelevant.

In Fig. 4, the solid (dashed) lines denote quantities for which only the polar (equatorial) solid angles have been taken into account for the corresponding volume- or surface-integration. At early times of $t \lesssim 0.2-0.3 \mathrm{~d}$, when the photosphere is still located at high velocities of $v \gtrsim 0.5 c$, the composition is nearly independent of the polar angle and material is lanthanide-rich in both polar and equatorial directions (see Fig. 2). Once the photosphere travels to slower ejecta mass shells, with $v \lesssim 0.5 c$, the composition gradient along the polar 

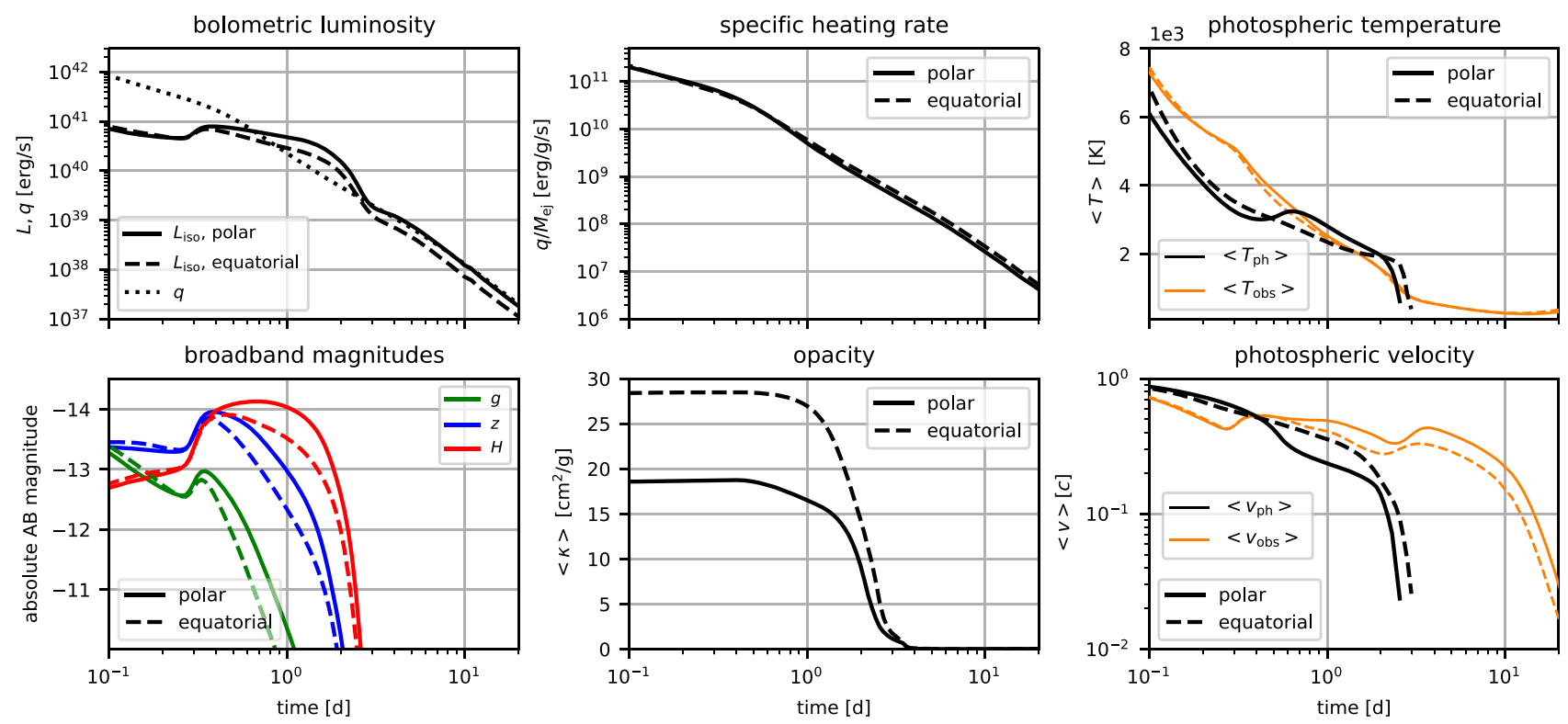

Figure 4. Global properties as functions of time characterizing the kilonova for the fiducial model DD2-135135. The left column shows in the top panel the bolometric isotropic-equivalent luminosity (cf. equation (21) together with the thermalized heating rate (cf. equation 25) and in the bottom panel absolute $\mathrm{AB}$ magnitudes (cf. equation 23), the middle column displays the mass-averaged specific heating rate (top) and mass-averaged opacity (cf. equation (27); bottom), and the right column provides in the top panel surface averages of the photospheric temperature (cf. equation (28); top) and photospheric velocity (cf. equation (29); bottom). Orange lines refer to the estimates of the photospheric temperature and velocity, $T_{\text {obs }}$ and $v_{\text {obs }}$, respectively, based on spectral fits to a blackbody spectrum (cf. equation (24). Solid (dashed) lines denote volume averages $(q,\langle\kappa\rangle)$ or surface averages (remaining quantities) over the polar (equatorial) domain.

angle, mentioned in Section 3.1, starts to become relevant. The lower opacities (cf. bottom middle panel in Fig. 4) together with the reduced mass loading in the polar region allow the photosphere to reach lower velocities and higher temperatures in the polar compared to the equatorial ejecta for a given time. The fact that due to the angular composition gradient radiation is released from polar ejecta more readily than from equatorial ejecta, provides one reason for the excess of polar compared to equatorial values of $L_{\text {iso }}$ at times $t \gtrsim 0.3 \mathrm{~d}$. Another reason, which is also observed for all considered models, is the oblate geometry of the photosphere at times $t \sim 1 \mathrm{~d}$, which results in a larger projected surface area when viewed face-on compared to edge-on (Wollaeger et al. 2018; Darbha \& Kasen 2020). On the other hand, angular variations of the specific radioactive heating rate do not seem to play a significant role for producing pole-to-equator emission asymmetries, as is suggested by the top middle panel in Fig. 4 (see Part I for an in-depth discussion of the heating rate as function of polar angle and velocity).

The orange lines in the right-hand panels of Fig. 4 also show, for comparison to the corresponding quantities measured at the photosphere, the spectral temperature, $\left\langle T_{\mathrm{obs}}\right\rangle$, and velocity, $\left\langle v_{\mathrm{obs}}\right\rangle$, inferred from the fluxes assuming blackbody emission. The agreement between both types of quantities is good, though not perfect, at least around peak emission times of $0.7 \mathrm{~d} \lesssim t \lesssim 3 \mathrm{~d}$. At earlier times, the spectral temperature (velocity) based on the blackbody model systematically overestimates (underestimates) the corresponding photospheric quantity. At late times, $t \gtrsim 3 \mathrm{~d},\left\langle T_{\text {obs }}\right\rangle$ and $\left\langle v_{\text {obs }}\right\rangle$ do not characterize the photospheric conditions anymore, since by then the photosphere has disappeared.

Looking at magnitudes in given frequency bands (cf. bottom left panel of Fig. 4), the kilonova of the dynamical ejecta peaks near the red end of the near-IR band, represented by $H$ in our case, and it does so in most of our models. The $H$-band magnitude near peak epoch is,
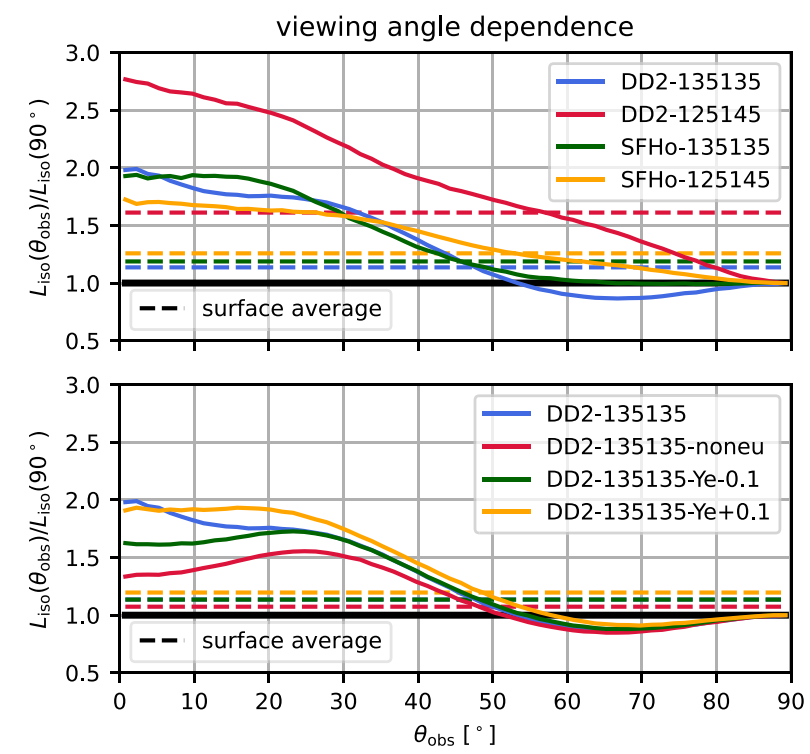

Figure 5. Isotropic-equivalent luminosity as a function of the inclination angle between rotation axis and observer line-of-sight, $\theta_{\mathrm{obs}}$, at $t=1 \mathrm{~d}$ normalized to the value at $\theta_{\mathrm{obs}}=90^{\circ}$ (solid lines). Dashed lines show the corresponding surface averages.

also roughly representative of all our models, $\sim-14$ mag and about 0.5 mag higher for polar observers than for equatorial ones.

The dependence of $L_{\text {iso }}$ on the observer angle $\theta_{\text {obs }}$ is shown at $t=1 \mathrm{~d}$ in Fig. 5. The pole-to-equator ratio of $L_{\text {iso }}$ is close to 2 for this model and similar to other models with a comparable semimajor 

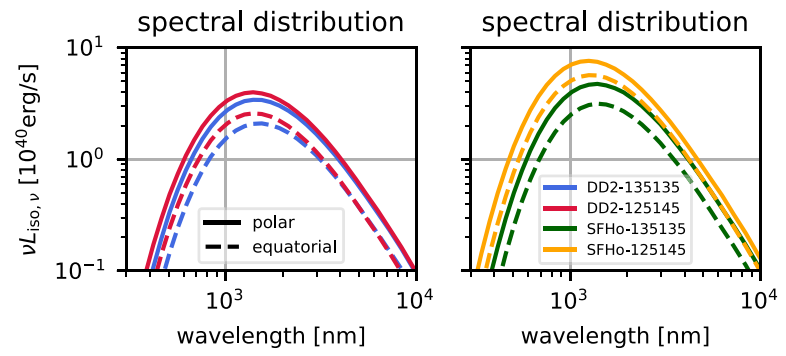

Figure 6. Monochromatic, isotropic-equivalent luminosity times frequency, $v L_{\text {iso, }}$, as a function of wavelength for the four models mentioned in the right-hand panel. Solid (dashed) lines denote surface averages over the polar (equatorial) domain.

axial ratio of the photophere (cp. black lines in Fig. 2). Model DD2125145 exhibits a higher value close to 3 , which is likely the result of a significantly more oblate ejecta geometry compared to the other models. Finally, the behaviour of the spectral distribution of $v L_{\text {iso, } v}$, plotted in Fig. 6, does not seem to exhibit a strong dependence on the observer angle.

\subsection{Model dependence}

In the following we examine how the previously identified properties change when switching the nuclear EoS, decreasing the mass ratio of the NS binary, ignoring neutrino interactions, and artificially reducing or enhancing the electron fraction $Y_{e}$ by 0.1 .

\subsubsection{Equation of state and binary mass ratio}

We show in Fig. 7 a similar selection of quantities as in Fig. 4, but now for the four models DD2-135135, DD2-125145, SFHo-135135, and SFHo-125145 and not distinguishing between polar and equatorial regions in the computation of the corresponding quantities. As shown in Part I, the abundance pattern of nucleosynthesis yields differs only by moderate amounts between these models and, hence, the initial opacities (cf. bottom middle panel of Fig. 7), as well as the specific heating rates (see fig. 8 in Part I) are rather similar for all models. The observed differences in the kilonova emission must therefore be connected to variations of the ejecta masses and velocities. Consistent with previous studies (e.g. Bauswein et al. 2013; Hotokezaka et al 2013; Radice et al. 2018b), the ejecta mass and average velocity increase for the softer SFHo EoS compared to the DD2 EoS, and the ejecta mass increases as well when reducing the mass ratio of the stellar binary. Indeed, the bolometric luminosities of the four models are roughly consistent with the order corresponding to the ejecta masses, at least in the late, optically thin phase of evolution, during which the luminosity is essentially given by the thermal heating power,

$L \approx L_{\text {thin }} \equiv\left\langle Q_{\text {heat }}\right\rangle M_{\mathrm{ej}}=q$.

Deviations from an exactly linear proportionality between $L_{\text {thin }}$ and $M_{\mathrm{ej}}$ at a given time $t$ are mainly connected to the thermalization efficiency, $f_{\text {th }}$, which grows with $M_{\mathrm{ej}}$ and decreases with the ejecta velocity, $\langle v\rangle$ (cf. Fig. 1). In contrast to the behaviour at late times, the luminosity differences right around the peak epochs are significantly smaller. The reason is that more massive ejecta take longer to become optically thin than less massive ejecta, namely roughly until

$t_{\text {peak }} \propto M_{\mathrm{ej}}^{\frac{1}{2}}\langle v\rangle^{-\frac{1}{2}}\langle\kappa\rangle^{\frac{1}{2}}$ as estimated based on the simplified case of a uniform distribution of density and opacity (e.g. Arnett 1982; Metzger et al. 2010; Grossman et al. 2014). Hence, since the radioactive heating rate declines approximately as

$\left\langle Q_{\text {heat }}\right\rangle \approx\left\langle f_{\text {th }}\right\rangle \times Q_{0} \times\left(\frac{t}{1 \mathrm{~d}}\right)^{-\alpha}$

(where $Q_{0} \approx 2 \times 10^{10} \mathrm{erg} \mathrm{g}^{-1} \mathrm{~s}^{-1}$ and $\alpha \approx 1.3$ reproduce well the total radioactive energy release rate, $Q+Q_{\text {neu }}$, resulting from our nucleosynthesis calculations; see Part $\mathrm{I}^{10}$ ), the luminosity at peak epoch scales only weakly with the ejecta mass (e.g. Grossman et al. 2014; Kasen et al. 2017; Wollaeger et al. 2018):

$$
\begin{aligned}
L_{\text {peak }} & \approx L_{\text {thin }}\left(t_{\text {peak }}\right) \\
& \propto Q_{0}\left\langle f_{\text {th }}\right\rangle_{\text {peak }} M_{\mathrm{ej}}^{0.35}\langle v\rangle^{0.65}\langle\kappa\rangle^{-0.65} .
\end{aligned}
$$

Assuming that the implicit dependence of the thermalization efficiency at peak, $\left\langle f_{\text {th }}\right\rangle_{\text {peak }} \equiv\left\langle f_{\text {th }}\right\rangle\left(t_{\text {peak }}\right)$, on $M_{\text {ej }}$ and $\langle v\rangle$ is subdominant, ${ }^{11}$ the behaviour of $t_{\text {peak }}$ and $L_{\text {peak }}$ in our models (cf. Table 1) can be understood reasonably well by means of equations (31) and (33), respectively.

In Table 1 we also provide the peak photospheric temperatures, $\left\langle T_{\mathrm{ph}}\right\rangle_{\text {peak }}$, and find that they all lie rather close to each other within $2.2 \ldots 2.7 \times 10^{3} \mathrm{~K}$, i.e. neither very sensitive to the nuclear EoS nor to the binary mass ratio. The weak dependence on $M_{\mathrm{ej}}$ and $\langle v\rangle$, is in agreement with expectations from an analytical estimate of the peak photospheric temperature, which can be derived using the StefanBoltzmann law under the assumption that the ejecta emit black-body radiation from their surface at about $r \approx\langle v\rangle t$ (e.g. Grossman et al. 2014):

$$
\begin{aligned}
\left\langle T_{\text {ph }}\right\rangle_{\text {peak }} & \approx\left[\frac{L_{\text {peak }}}{\sigma_{\mathrm{SB}} 4 \pi\left(\langle v\rangle t_{\text {peak }}\right)^{2}}\right]^{\frac{1}{4}} \\
& \propto Q_{0}^{0.25}\left\langle f_{\text {th }}\right\rangle_{\text {peak }}^{0.25} M_{\mathrm{ej}}^{-0.16}\langle v\rangle^{-0.09}\langle\kappa\rangle^{-0.41}
\end{aligned}
$$

(where $\sigma_{\mathrm{SB}}$ is the Stefan-Boltzmann constant). Measured in terms of the power by which they determine $\left\langle T_{\mathrm{ph}}\right\rangle_{\text {peak }}$ in this simplified analytical estimate, the mass and velocity of the ejecta are less important than the opacity and heating rate. Not surprisingly, considering the rather similar values of $\left\langle T_{\text {ph }}\right\rangle_{\text {peak }}$, all models peak in the same $(H)$ frequency band.

In summary, in our kilonova models the luminosity (spectral temperature) is rather sensitive (insensitive) to variations of the nuclear EoS and the binary mass ratio. Since the composition is fairly independent of the EoS and mass ratio, the bolometric luminosity is therefore mainly determined by the ejecta mass and velocity. We note that the simplified grey opacities used here are not suited for an indepth analysis of spectral features. More sophisticated calculations using opacities based on atomic lines may reveal sensitivities that are obscured by our scheme.

\subsubsection{Ignoring neutrino interactions}

The impact of neutrino interactions on the composition and the kilonova of dynamical ejecta (as well as other ejecta components)

\footnotetext{
${ }^{10}$ Note that the analytic fit presented in Part I with a scaling factor of $1 \times$ $10^{10} \mathrm{erg} \mathrm{g}^{-1} \mathrm{~s}^{-1}$ was for the heating rate without neutrino contributions, $Q$, whereas here we consider $Q+Q_{\text {neu }}$.

${ }^{11}$ We stress that $\left\langle f_{\text {th }}\right\rangle\left(t_{\text {peak }}\right)$ varies much less between individual models than $\left\langle f_{\text {th }}\right\rangle(t)$ at some fixed time, $t$, as can be seen from Fig. 1 using the peak times provided in Table 1.
} 

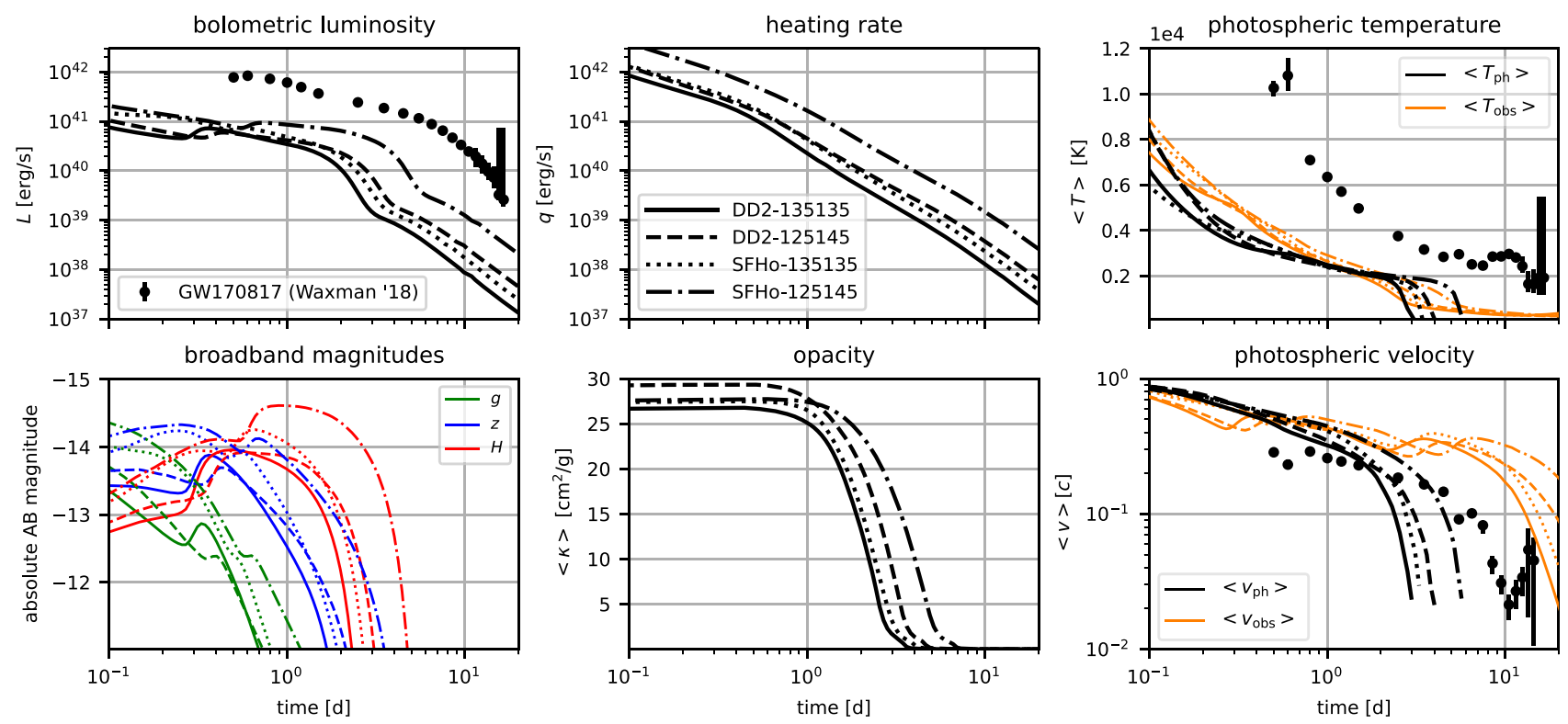

Figure 7. Same as Fig. 4 but comparing the four hydrodynamic models with different nuclear EoSs and binary mass ratios and not distinguishing between polar and equatorial regions, i.e. only spherically averaged or integrated quantities are shown. Note that the top middle panel now shows $q$ instead of $q / M_{\mathrm{ej}}$.

represents one of the most uncertain and challenging aspects in theoretical multimessenger modelling of NS mergers given the difficulties of solving the Boltzmann equation for neutrinos (e.g. Wanajo et al. 2014; Goriely et al. 2015; Foucart et al. 2018; Martin et al. 2018; Radice et al. 2018b; Sumiyoshi et al. 2021). Until a few years ago, most merger simulations therefore either completely neglected neutrino interactions or implemented them in a way that neglects net neutrino absorption and/or the advection and equilibration of trapped neutrinos. The advection of trapped neutrinos can be accounted for without significant efforts and even without computing any neutrino interaction rates - basically by replacing the electron fraction with the total lepton fraction in optically thick regions (e.g. Goriely et al. 2015; Ardevol-Pulpillo et al. 2019). However, the main challenge lies in the reliable description of the emission and absorption rates in the semitransparent regions surrounding the hot merger remnant once it is formed. In order to quantify the impact of (not) including any neutrino interactions after the first touch of the two stars, we introduced in Part I a variation of model DD2-135135 (called DD2135135-noneu here), in which $Y_{e}$ is held constant after the point when the two stars plunge into each other. In this model, the abundances of elements with mass numbers $90 \lesssim A \lesssim 140$ are found to be reduced, while the mass fractions of lanthanides and heavier elements are enhanced by a factor of 2-3. Moreover, the pole-to-equator composition gradient basically disappeared and high values of $X_{\mathrm{LA}}$ $\gtrsim 0.1$ are now found at all latitudes (see fifth row of Fig. 2). For the heating rate, the lack of low-mass elements causes a reduction at early times $(0.1 \mathrm{~d} \lesssim t \lesssim 1 \mathrm{~d})$, while the excess of heavy elements produces an increase at late times $(1 \mathrm{~d} \lesssim t \lesssim 100 \mathrm{~d})$ compared to the full neutrino model (cf. section 3.2 and fig. 8 of Part I).

Fig. 8 provides insight about the extent to which the kilonova light curve and related quantities are affected by neglecting neutrino interactions during (most of) the merger process. Initially, when the temperatures are above the recombination temperatures, the enhancement factor of $\sim 2$ in the lanthanide fraction translates into an opacity increase of $\approx 20$ per cent. The weaker radioactive heating under more opaque conditions at $t<1 \mathrm{~d}$ explains why the emission peak is fainter by about $30-50 \$$ per cent and is delayed by $\sim 10$ per cent (cf. top left panel of Fig. 8 and Table 1). At late times, when opacity effects have ceased and heavier elements dominate the heating rate, the situation is reversed because of the higher abundance of heavier elements in the 'noneu' model. Considering broadband light curves, the kilonova peak remains in the red regime of the near-IR band, represented here by the $H$-band, while its magnitude is reduced by about $0.3 \mathrm{mag}$. The photospheric temperatures near peak epoch are reduced, though only mildly.

\subsubsection{Sensitivity to $\mathrm{Y}_{\mathrm{e}}$}

An additional sensitivity test to bracket the uncertainties in the treatment of weak interactions can be conducted by manually varying the electron fraction at the onset of the nuclear network calculations, $Y_{e}\left(\rho_{\text {net }}\right)$. To this end, we set up two models that are identical to the fiducial model but where $Y_{e}$ of all trajectories is increased and reduced, respectively, by 0.1 (with a lower limit of 0.005 imposed). The nucleosynthesis yields of these models have already been discussed in Part I, see Section 3.3 and Fig. 11 therein.

In Fig. 9, we compare both models with the fiducial model. Artificially reducing $Y_{e}$ by 0.1 yields results that are very similar to the case of completely neglecting neutrino interactions (see previous section and cf. Fig. 8), namely a delayed and fainter peak. This is not surprising considering that the average electron fractions of the corresponding models are comparable $\left(\left\langle Y_{e}\right\rangle \approx 0.17\right.$ and 0.13 , respectively; cf. Table 1). In the opposite case of increasing $Y_{e}$ by 0.1 , the mass fraction of lanthanides and the opacity are reduced roughly by factors of 4 and 2, respectively. The lower opacities result in a significantly shorter duration of the kilonova and a slightly more luminous peak. The luminosity enhancement due to the reduced opacity is, however, partially compensated by the weaker radioactive heating rates in the $Y_{e}+0.1$ model. At $t=1 \mathrm{~d}$, for instance, the heating rate is about $q /\left(10^{40} \mathrm{erg} \mathrm{s}^{-1}\right)=1.66$ compared to 2.32 in the reference model. The reduced opacities also shift the spectral peak to the blue end of the near-IR domain, as can be seen by the enhanced 

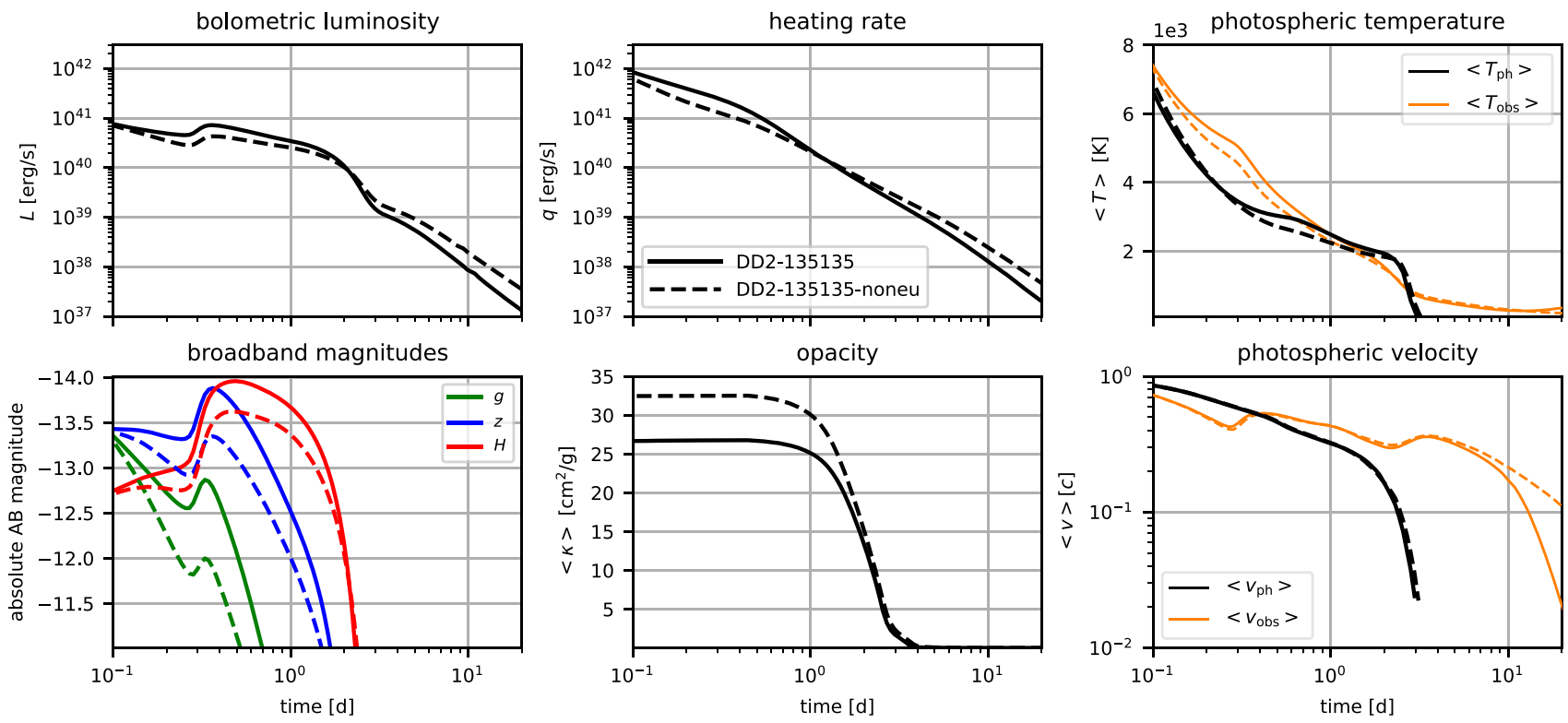

Figure 8. Same as Fig. 7 but comparing the fiducial model DD2-135135 with its counterpart DD2-135135-noneu, in which neutrino reactions have been neglected after the plunge.
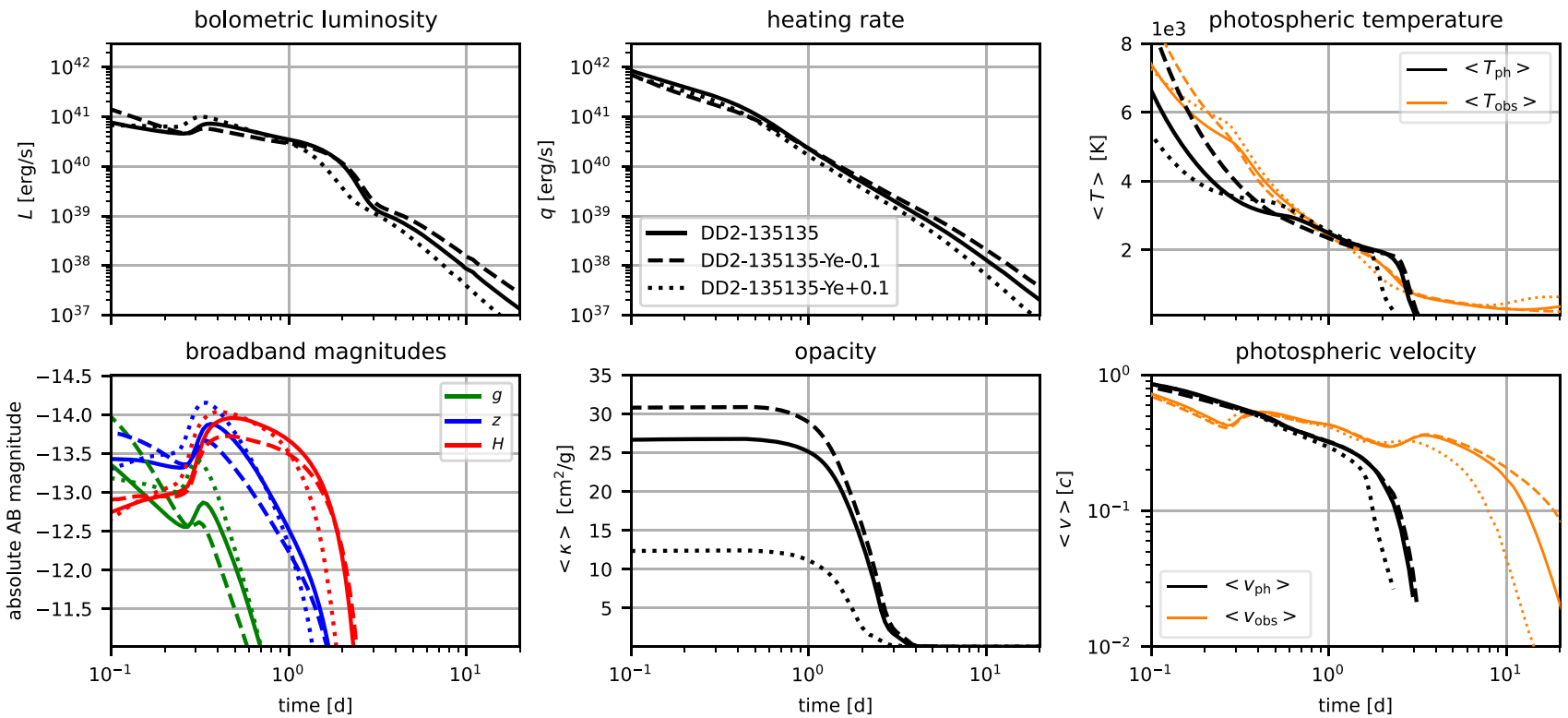

Figure 9. Same as Fig. 7 but comparing the fiducial model DD2-135135 with its counterparts DD2-135135-Ye-0.1 and DD2-135135-Ye + 0.1, in which the electron fraction, $Y_{e}$, was shifted by $-0.1(+0.1)$ for all trajectories.

$z$-band magnitudes in the lower left panel of Fig. 9. Yet, despite the considerable shift of $Y_{e}$ and resulting reduction of the lanthanide fraction down to $\left\langle X_{\mathrm{LA}}\right\rangle \approx 0.02$, the emission in the visible bands is still subdominant compared to that in the near-IR domain, i.e. the transient would classify as a red kilonova.

Summarizing the results of Sections 3.3.2 and 3.3.3: Differences in the treatment of neutrino effects can induce variations in brightness of up to a factor of 2 and in duration of $\sim 20$ per cent, i.e. an amount comparable to that resulting from changing the binary mass ratios or the nuclear EoSs.

\section{DISCUSSION}

\subsection{Role of dynamical ejecta in AT2017gfo/GW170817}

A comprehensive understanding of the origin of each component in the kilonova accompanying GW170817, AT2017gfo, is still elusive. Although the lack of the secular post-merger ejecta in our study and the small set of considered EoSs and NS merger configurations defies a conclusive comparison, we can nevertheless briefly speculate whether our models, which in contrast to most previous models are based on consistent nucleosynthesis trajectories from hydrody- 
namical simulations, would, or not, support the possibility that the dynamical ejecta alone are responsible for either of the two, red or the blue, kilonova components. The observed bolometric luminosity of AT2017gfo, as constructed by Waxman et al. (2018), is shown by filled circles in Fig. 7, as well as estimates of the spectral temperature and photospheric velocity, which can be compared directly to $\left\langle T_{\mathrm{obs}}\right\rangle$ and $\left\langle v_{\text {obs }}\right\rangle$ (orange lines in the right-hand panels of Fig. 7). As can be seen, none of our models appears to be compatible with either component of AT2017gfo.

The early, blue component of AT2017gfo, i.e. the emission observed until $t \sim 1-1.5 \mathrm{~d}$ (e.g. Nicholl et al. 2017), exhibited a bolometric luminosity of almost one order of magnitude higher than the surface-averaged values predicted by our models, and its spectral temperatures were roughly twice as high as in our models. The combination of both discrepancies, too faint luminosities and too red colours, could hardly be resolved by just increasing the ejecta mass, because more massive ejecta would rather reduce the peak temperatures, as suggested by equation (34) neglecting uncertainties connected to the thermalization efficiency. The comparison suggests that, in agreement with previous studies based on more simplified one-zone models (Villar et al. 2017), significantly lower lanthanide mass fractions, and corresponding opacities, may be required to reproduce the early component. Even though the average electron fraction is raised significantly as a result of a sophisticated inclusion of neutrinos in our models (e.g. from $\left\langle Y_{e}\right\rangle=0.13$ to 0.27 for model DD2-135135; cf. Table 1), the amount of lanthanides still seems to be too high to produce an optical transient like the one in AT2017gfo. However, our set of investigated models is rather small, and more exhaustive explorations using other EoSs and NS binary configurations compatible with GW170817 are needed to clarify whether cases exist in which the lanthanide mass fraction becomes significantly smaller than observed for our models.

The relatively red spectral colours seen in the kilonova signals of our models suggests a better agreement of our models with the red component of AT2017gfo (e.g. Chornock et al. 2017), ${ }^{12}$ especially keeping in mind that (cf. Fig. 5) isotropic luminosities observed from polar viewing angles can be enhanced by some factor compared to the surface-averaged isotropic luminosities plotted in Fig. 7. However, the ejected mass would presumably need to be significantly larger (by a factor of a few) than the $0.009 \mathrm{M}_{\odot}$ of our most massive model in order for the peak to occur sufficiently late and luminous (cp. Fig. 7 and equations 31 and 33). This could possibly be achieved by very asymmetric cases and a soft EoS (e.g. Bauswein et al. 2013; Sekiguchi et al. 2016; Radice et al. 2018b). Moreover, a crucial role may be played by the uncertainties associated with the thermalization efficiency, $f_{\text {th }}$ (Barnes et al. 2016; Kasen \& Barnes 2019; Wu et al. 2019). The fact that $f_{\text {th }}$ increases with the ejecta mass could improve the prospects in this regard.

In any case, our analysis remains incomplete and inconclusive, because we only consider a small set of EoSs and binary configurations and we only include a single ejecta component. A more realistic model of AT2017gfo would need to account for genuine multicomponent effects, such as photon transport from one ejecta component to another (e.g. Kawaguchi et al. 2018), or photon

\footnotetext{
${ }^{12} \mathrm{We}$ remark that post-merger disc outflows are often considered more suitable to explain the red component (e.g. Kasen et al. 2017), because they are typically more massive than the dynamical ejecta while carrying comparable lanthanide fractions (e.g. Just et al. 2015a; Wu et al. 2016; Siegel \& Metzger 2017; Just et al. 2022). See e.g. Tanaka et al. (2017), Perego et al. (2017), and Kawaguchi et al. (2018) for the discussion of alternative scenarios.
}

blocking of one component by another (often called 'lanthanide curtain'; see e.g. Kasen, Fernández \& Metzger 2015; Korobkin et al. 2021).

\subsection{Importance of consistent ejecta sampling}

An often employed approximation in modelling ejecta and kilonovae from NS mergers is to assume representative bulk values of the electron fraction, expansion velocity, and entropy for the entire ejecta, or for sub-domains within the ejecta, and to derive from those the composition, heating rate, and opacity using a single nucleosynthesis calculation. Obviously, such single-trajectory models carry some uncertainty, because in reality the ejecta exhibit a broad spectrum of hydrodynamic and thermodynamic properties, which may result in large variations of heavy-element yields among different fluid elements in the ejecta. The yields obtained by averaging over a collection of nucleosynthesis trajectories may predict results that cannot be accounted for by a single nucleosynthesis calculation along a trajectory of averaged fluid properties. Our method of detailed ejecta sampling by post-processing individual outflow trajectories using consistent hydrodynamic data allows to test the uncertainty of single-trajectory models by comparing the average opacities and heating rates predicted by either approach.

We first consider the opacity. It is well known (e.g. Hoffman, Woosley \& Qian 1997; Ruffert et al. 1997; Lippuner \& Roberts 2015; Lemaître et al. 2021) that the production of lanthanides along a typical outflow trajectory from NS mergers becomes inefficient as soon as the electron fraction exceeds a threshold value, $Y_{e}^{\text {crit }}$, which, for typical merger outflows and mainly depending on the entropy per baryon, lies within $Y_{e}^{\text {crit }}=0.25-0.3$. As can be seen by the dots in the middle panel of Fig. 10, this behaviour is reproduced well by nucleosynthesis yields for individual ejecta trajectories of our model DD2-135135: All trajectories with $Y_{e} \gtrsim 0.28$ exhibit extremely low lanthanide fractions of $X_{\mathrm{LA}}<10^{-4}$. This step-like behaviour is often used to characterize also the bulk ejecta or sub-regions of the ejecta by assigning very low values of $X_{\mathrm{LA}}$ to regions with average $Y_{e}>Y_{e}^{\text {crit }}$. However, if instead of individual trajectories we consider finite subdomains of the ejecta, for example obtained by dividing the Northern hemisphere into 30 angular bins (see blue crosses in Fig. 10), we receive quite a different relation between $Y_{e}$ and $X_{\mathrm{LA}}$. Due to mixing of low- $X_{\mathrm{LA}}$ fluid elements with high- $X_{\mathrm{LA}}$ fluid elements, the sharp cut-off is replaced by a much more shallow decline, such that regions with average $Y_{e}>0.4$ can still contain a high fraction of lanthanides $X_{\mathrm{LA}}>0.01$ and possess a correspondingly high opacity. If, for instance, we had estimated the opacity of angular bins based on the $\kappa_{\mathrm{LA}}\left(Y_{e}\right)$ behaviour of individual trajectories, e.g. by using $\kappa_{\mathrm{LA}}\left(Y_{e}>\right.$ $0.28)=0.2$ and $\kappa_{\mathrm{LA}}\left(Y_{e}<0.28\right)=30 \mathrm{~cm}^{-2} \mathrm{~g}^{-1}$, the average opacity of the polar $(\theta<\pi / 4)$ ejecta would be $0.2 \mathrm{~cm}^{-2} \mathrm{~g}^{-1}$, i.e. grossly underestimated compared to the value of $\approx 6.2 \mathrm{~cm}^{2} \mathrm{~g}^{-1}$ that would result if we had used the same $\kappa_{\mathrm{LA}}\left(Y_{e}\right)$ function for individual particles instead. Hence, approximating $X_{\mathrm{LA}}$ or $\kappa_{\mathrm{LA}}$ based on the average $Y_{e}$ of an ensemble of trajectories can lead to a significant underestimation of $X_{\mathrm{LA}}$ or $\kappa_{\mathrm{LA}}$, which tends to be more serious for a higher degree of inhomogeneity of the ejecta. While here we consider only the dynamical ejecta, this aspect is likely to be relevant also for turbulence-driven secular ejecta, which typically exhibit a broad spectrum of $Y_{e}$ (e.g. Fernández \& Metzger 2013; Siegel \& Metzger 2017; Just et al. 2022), while it might be less relevant for neutrino-driven winds, which often have a rather smooth structure (e.g. Perego et al. 2014; Fujibayashi et al. 2018).

Next we take a look at the radioactive heating rates. As is already clear from the analytic scaling laws for $L_{\text {thin }}$ and $L_{\text {peak }}$ (cf. 

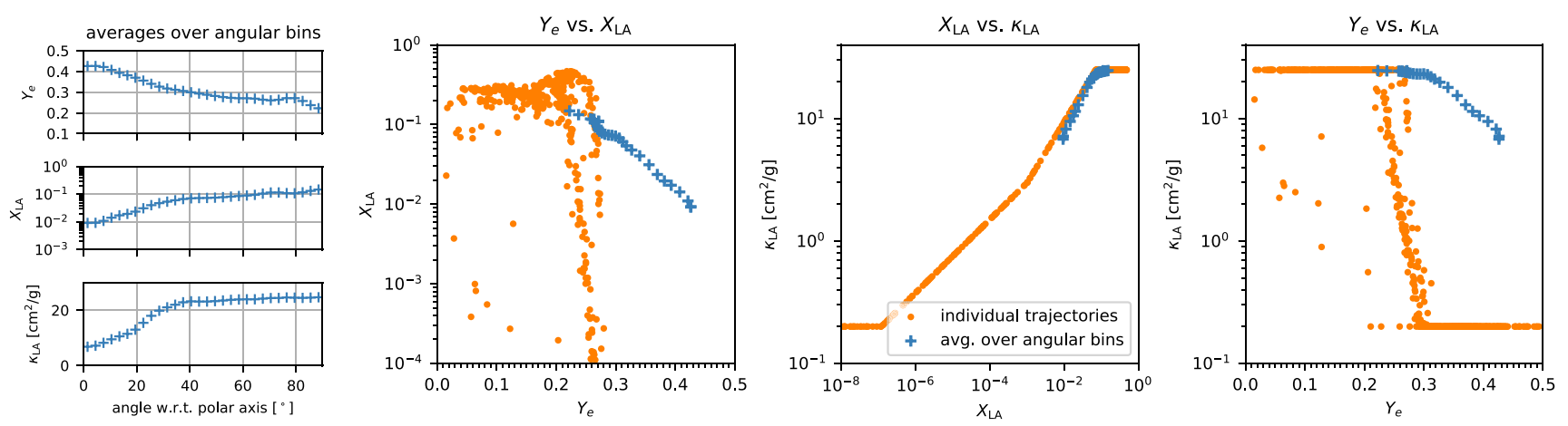

Figure 10. Example illustrating the discrepancy between correlations obtained for individual trajectories and correlations between averages over finite domains. All plotted data are taken from model DD2-135135. Three left plots: mass-averages of the electron fraction, $Y_{e}$, lanthanide mass fraction, $X_{\mathrm{LA}}$, and (temperatureindependent part of the) opacity, $\kappa_{\mathrm{LA}}$, over the volumes defined by 30 equidistant polar-angle bins dividing the Northern hemisphere. Three right plots: pair-wise correlation plots between the same data as in the left plots. For comparison, orange dots denote the corresponding data measured for individual outflow trajectories. A sharp cut-off of $X_{\mathrm{LA}}$ around $Y_{e} \sim 0.28$ is only visible for single-trajectory data, while much higher values of $X_{\mathrm{LA}}\left(Y_{e}\right)$, and therefore of $\kappa_{\mathrm{LA}}\left(Y_{e}\right)$, can be reached when considering collections of trajectories in finite regions.

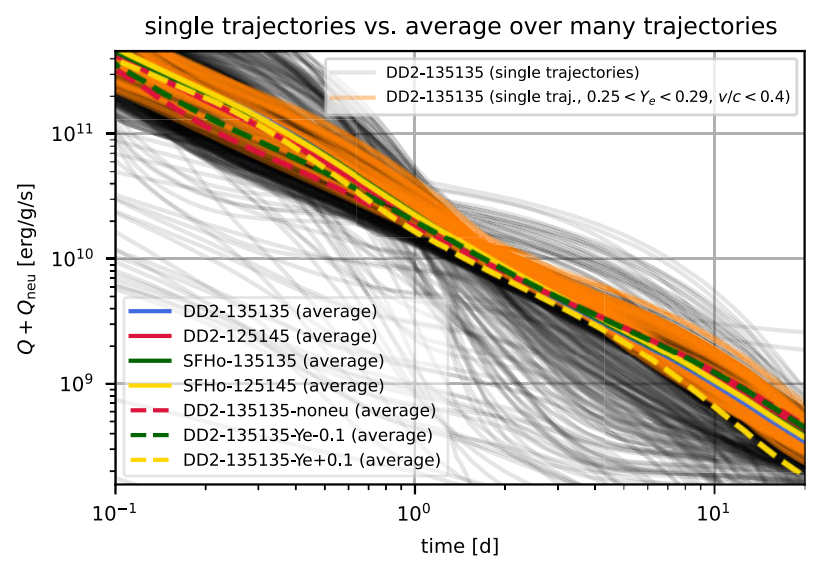

Figure 11. Comparison of total radioactive energy-release rates, $Q+$ $Q_{\text {neu }}$, along individual outflow trajectories of model DD2-135135 and the corresponding rates mass averaged over all trajectories of a given model. Orange lines denote the subset of particles of model DD2-135135 with $Y_{e}$ close to the average value of $\left\langle Y_{e}\right\rangle=0.27$ and with velocities $v / c<0.4$. Values of $Q+Q_{\text {neu }}$ differ more strongly between individual trajectories than between ensemble averages of different models, even for models with rather diverse values of $0.13<\left\langle Y_{e}\right\rangle<0.37$.

equations (30) and (33), respectively) a reliable prediction of the heating rate is mandatory for any kilonova model to properly infer the ejecta mass from observations during the peak or the optically thin phase (e.g. Rosswog et al. 2018; Wanajo 2018; Wu et al. 2019; Barnes et al. 2021). For simplicity, we now ignore the additional complexity connected to the thermalization efficiency and assume $f_{\text {th }}=1$. For the heating rates, the analysis is not as straightforward as for the opacities, where the strong non-linearity of $X_{\mathrm{LA}}\left(Y_{e}\right)$ represents the main culprit, because the heating rates are determined by decay chains involving a large number of isotopes, of which the abundances are quite sensitive also to the entropy and expansion time-scale. Nevertheless, we can get a basic idea of the uncertainty of singletrajectory models by comparing the heating rates for individual trajectories with the average heating rates. To that end, in Fig. 11 we plot, apart from the global heating rates for each model, the heating rates of all particles of model DD2-135135 using black, slightly transparent lines, while orange lines are used to denote only the subset of these particles that have $Y_{e}$ close to the average value of $\left\langle Y_{e}\right\rangle \approx 0.27$ and velocities of $v / c<0.4$. Even for this subset of presumably representative trajectories (in the sense that $Y_{e}$ and $v$ are in the ballpark of expected bulk values), the range of variation of the heating rates is substantial and amounts to factors of 2-4 during the entire period of $0.1 \mathrm{~d}<t<20 \mathrm{~d}$ that is relevant for the kilonova emission. On the other hand, the substantially smaller model-bymodel variations of the average heating rates (thick lines in Fig. 11) indicate that the average heating rate of ensembles of trajectories are subject to a much reduced level of randomness than the heating rate of individual particles. This finding is particularly remarkable, because we even consider models with artificially changed values of $Y_{e}$ that span a large range of values for $\left\langle Y_{e}\right\rangle$ between 0.13 and 0.37 (cf. Table 1).

From the above discussions, we conclude that predictions for the nucleosynthesis yields and the corresponding kilonova signal that are based on one-zone models or single-trajectory modelling may carry substantial systematic uncertainties in cases where the thermodynamic properties are not homogeneous throughout the ejecta but given by a broad distribution.

\subsection{Comparison with previous studies}

We will now briefly compare some of our results with previous studies. The level of sophistication of kilonova models is growing quickly, and many studies employ their own ways of dealing with the large number of required physics ingredients. Moreover, given the large parameter space, it is difficult to find studies that discuss models with a match of the basic characteristic parameters (geometry, mass, velocity, composition, etc.), which would be required for a meaningful quantitative comparison. Hence, we will only compare a few elementary features with selected studies.

While the number of kilonova studies directly based on the outputs from hydrodynamical simulations is still very small, quite a few studies exist by now that investigate multidimensional kilonovae based on manually constructed ejecta configurations with homogeneous distributions of the heating rate and opacity (e.g. Wollaeger et al. 2018; Barbieri et al. 2019; Bulla 2019; Darbha \& Kasen 2020; Kawaguchi et al. 2020; Heinzel et al. 2021; Korobkin et al. 2021). Darbha \& Kasen (2020) studied the emission characteristics as a function of the observer angle based on parametrized ellipsoids, tori, 
comparison with Korobkin +21
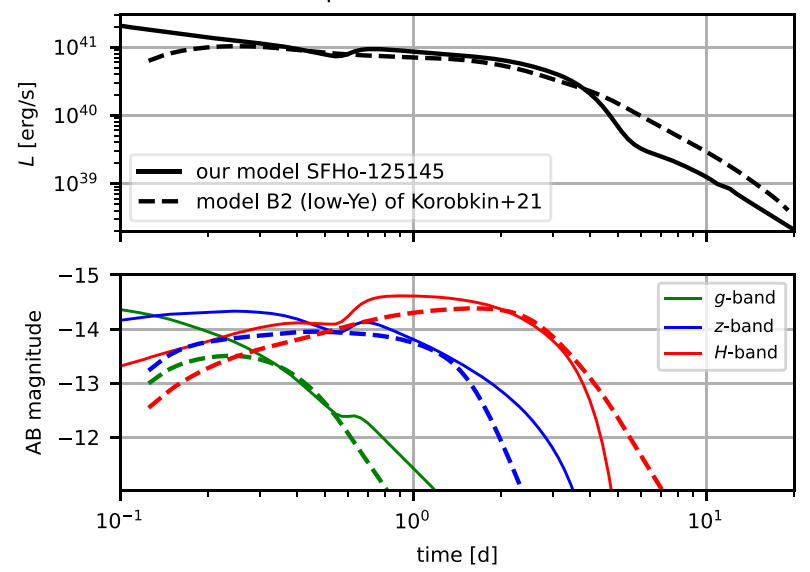

Figure 12. Exemplary comparison of the surface-integrated bolometric luminosity (top panel) and the $\mathrm{AB}$ magnitudes in selected bands (bottom panel) of model B2 (low- $Y_{e}$ version) of Korobkin et al. (2021) with our model SFHo-125145. The good agreement with Korobkin et al. (2021), who employ a Monte Carlo radiative-transfer scheme with atomic-physics based opacities supports the validity of our results obtained with an approximate radiative transfer solver with parametrized opacity treatment.

and conical sections with a grey and constant opacity and fitted the results with an analytic function for the ratio $L_{\text {iso }}\left(0^{\circ}\right) / L_{\text {iso }}\left(90^{\circ}\right)$ in dependence of the projected surface area of the emitting object. For an ellipsoid with a semimajor axial ratio of $\sim 1.5-2$, which to first order resembles the shape of the photospheres that we observe in our models (cf. Fig. 2), they predict values of $L_{\text {iso }}\left(0^{\circ}\right) / L_{\text {iso }}\left(90^{\circ}\right) \approx 1.5-3$ that are in good agreement with our results (see Fig. 5).

Various types of parametrized, axisymmetric ejecta configurations have been employed in a series of papers by the Los Alamos group (e.g. Wollaeger et al. 2018; Korobkin et al. 2021; Wollaeger et al. 2021) using atomic-physics-based opacities (Fontes et al. 2020) and two different choices for the composition (Even et al. 2020). The ellipsoidal geometry of photospheres found here for the dynamical ejecta is roughly reproduced by the biconcave ('B') configuration introduced by Korobkin et al. (2021), which however was dropped again in the set of models by Wollaeger et al. (2021). Out of the single-component models considered by Korobkin et al. (2021), their model B2, with an average velocity of $0.2 c$ and a mass of $0.01 \mathrm{M}_{\odot}$, possesses parameters with the closest agreement to one of our models, namely to SFHo-125145. A caveat for our comparison is, however, that their 'low- $Y_{e}$ ' case exhibits a lower lanthanide fraction $\left(X_{\mathrm{LA}}=0.032\right.$; see Even et al. 2020 and Korobkin et al. 2021) compared to our model (0.114). In Fig. 12, we compare ${ }^{13}$ the angleaveraged bolometric light curves and magnitudes for both models and find rather good agreement, both quantitatively and qualitatively regarding the property that the emission peaks in the same near-IR frequency regime.

A similar approach as in the aforementioned works was pursued in Kawaguchi et al. (2018, 2020), however, almost exclusively considering multicomponent (dynamical plus secular) ejecta models. Broadband magnitudes for two single-component models of dynamical ejecta, with masses of 0.003 and $0.01 \mathrm{M}_{\odot}$ and average velocity of $0.25 c$ are presented in appendix A of Kawaguchi et al. (2020).

\footnotetext{
${ }^{13}$ The data for model B2 was downloaded from https://ccsweb.lanl.gov/astr
} $\mathrm{o} /$ transient/transients_astro.html.
Despite the fact that their setup agrees fairly well with ours - the main difference is probably the more toroidally shaped ejecta distribution in their case - we find only modest agreement. Independent of the observer angle their kilonova is brighter by about 1 mag in the nearIR bands and, at early times and polar viewing angles, they obtain a considerably stronger optical component than us. The origin of these differences remains unclear but could be connected to the more advanced set of opacities (which are based on Tanaka et al. 2020) employed in these studies and to the different ejecta geometry.

Among the few available kilonova models that adopt ejecta properties directly from hydrodynamical simulations are Kawaguchi et al. (2021) and Radice et al. (2018b). A direct comparison with the light curves of Kawaguchi et al. (2021) is not possible, however, because they additionally follow the long-term evolution of the NSdisc remnant, which produces massive viscously-driven outflows that dominate the dynamical ejecta. Yet, a noteworthy difference to the models in Kawaguchi et al. (2021) is the prolate geometry of their total ejecta, which is in contrast to the rather oblate structures that we find for just the dynamical ejecta at peak epoch.

Radice et al. (2018b) reported kilonova light curves that are obtained by a superposition of quasi-spherically symmetric models adopting the formalism by Grossman et al. (2014), Martin et al. (2015), and Perego et al. (2017). Radice et al. (2018b) used data provided by binary NS merger simulations for the emission from the dynamical ejecta, while they employed a parametric wind model to estimate the contribution from neutrino- plus viscously-driven postmerger ejecta. Although no light curves just for the dynamical ejecta components are shown in Radice et al. (2018b), one can indirectly infer from their results that the dynamical ejecta produce significant emission in the $g$ and $z$ bands, with absolute AB magnitudes of about -15 mag (corresponding to apparent magnitudes of $\approx 18$ mag when observed at a distance of $40 \mathrm{Mpc}$ ) for dynamical ejecta masses of $\approx 0.003 \mathrm{M}_{\odot}$. Our models, which seem to broadly agree in the massand $Y_{e}$-distributions of the ejecta, instead produce significantly fainter emission which peaks in bands with lower frequency. One reason for the higher luminosities may be that Radice et al. (2018b) assigned the opacities as function of average $Y_{e}$ values along angular bins (see Section 4.2 for a discussion). Another, more likely explanation may come from the fact that Radice et al. (2018b) employed the extremely high heating rates by Perego et al. (2017). These rates, which are shown in Fig. 13, were not adopted from nucleosynthesis calculations but were calibrated such that the semi-analytic kilonova models by Perego et al. (2017) reproduce the observed kilonova AT2017gfo. ${ }^{14}$ Switching to such a high heating rate enhances the peak luminosities substantially, because the heating rate enters $L_{\text {peak }}$ linearly, whereas the ejecta mass, velocity, and opacity only enter with a lower power (cf. equation 33). Hence, we expect the heating rate to be the main reason for the observed discrepancies between their kilonova results and ours.

Although the high heating rates assumed in Perego et al. (2017) cannot be excluded entirely, to our knowledge no state-of-the-art network solver currently exists that produces heating rates of that magnitude for the thermodynamic conditions under consideration. A systematic discussion of the sensitivity of the heating rate with respect to nuclear-physics uncertainties is, however, deferred to a future study. We point out that Barnes et al. (2021) and Zhu et al. (2021) have recently taken a step in that direction and report large variations between $Q$ profiles with different nuclear physics

\footnotetext{
${ }^{14} \mathrm{~A}$ similar approach involving even higher heating rates was recently adopted by Breschi et al. (2021).
} 


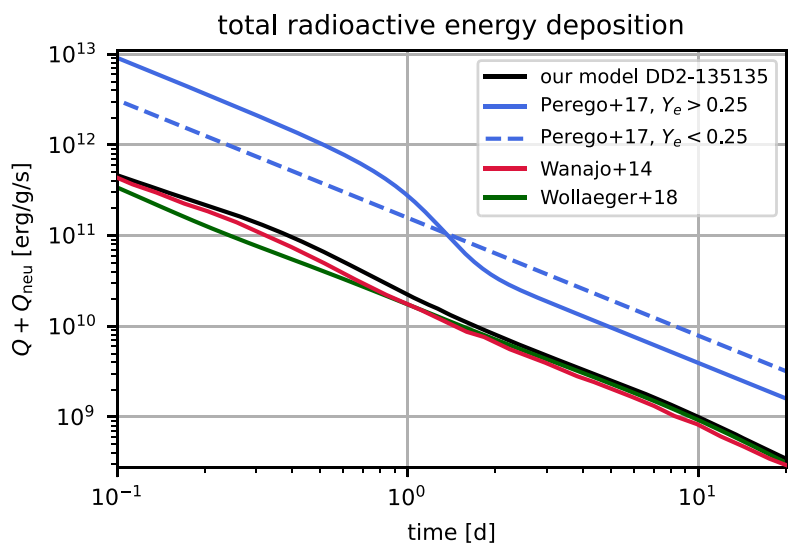

Figure 13. Comparison of mass-averaged total radioactive energy-release rates resulting from our nucleosynthesis calculations for model DD2-135135 with heating rates employed in other kilonova studies (not accounting for thermalization efficiencies). The red curve (extracted from fig. 5 of Wanajo et al. 2014) denotes the heating rate obtained from a nucleosynthesis analysis of a neutrino-hydrodynamical merger simulation and was employed for dynamical ejecta in, e.g. Kawaguchi et al. (2018), Kawaguchi, Shibata \& Tanaka (2020). The green line (extracted from fig. 4 of Wollaeger et al. 2018) is based on a network calculation along an exemplary outflow trajectory of model B of Rosswog et al. (2014) and was employed for dynamical ejecta in, e.g. Wollaeger et al. (2018), Korobkin et al. (2021), and Wollaeger et al. (2021). Blue lines (cf. equation 2 and case BF of Perego et al. 2017) represent the $Y_{e}$-dependent prescription employed by Perego et al. (2017) and Radice et al. (2018b), which is not based on a nuclear network calculation but deduced from a light-curve fit to AT2017gfo.

inputs. However, the range of uncertainty may not be as dramatic as suggested by these studies, because, first, some of their most extreme cases are produced by nuclear models, which are known to be inconsistent with currently known experimental data, ${ }^{15}$ and secondly, their analysis is based on single outflow trajectories instead of trajectory ensembles, which may artificially enhance the range of scatter as indicated by the comparison discussed in Section 4.2 (fig. 11 there).

\section{SUMMARY AND CONCLUSIONS}

In this study we applied a new methodical treatment to predict the kilonova emission of dynamical ejecta adopting tracer particles from general relativistic neutrino-hydrodynamics simulations. In contrast to many previous kilonova models using only the bulk properties of the ejecta, the tracer-particle approach samples the local, time-dependent properties of the ejected material, as well as the corresponding nucleosynthesis results for the radioactive heating rate, lanthanide mass fraction, and mean baryon number. The kilonova emission is computed by means of a two-moment transport scheme employing the approximate M1 closure and assuming a simplified, heuristic expression of the photon opacity as function of the lanthanide fraction and the temperature. We applied our approach to the dynamical ejecta of four different NS-merger configurations

${ }^{15}$ For example, Barnes et al. (2021) and Zhu et al. (2021) employ the SLy4 HFB masses, which are known to predict all experimental masses of eveneven nuclei with a root-mean-square deviation as large as $5 \mathrm{MeV}$ (Stoitsov et al. 2003). with different nuclear equations of state and different binary mass ratio. Moreover, in order to quantify the sensitivity with regard to uncertainties of weak interaction effects taking place during the dynamical merger phase, we compared with three additional cases with artificially modified electron fractions. Our results are summarized as follows:

(i) At times close to peak emission, all models exhibit photospheres that globally resemble ellipsoids with semimajor axial ratio of 1.2-2. The globally oblate geometry of the photosphere is a result of less efficient matter ejection towards the poles, as well as systematically higher electron fractions, and therefore reduced opacities, in the polar compared to equatorial regions. Due to the violent, highly dynamic matter-ejection processes during the merger, the photosphere is not smooth but rather corrugated and characterized by irregular structures that change with time as the photosphere travels deeper into the ejecta.

(ii) For the four dynamical ejecta models with regular treatment of weak interactions, masses between 0.002 and $0.009 \mathrm{M}_{\odot}$, average velocities of $\approx 0.25 c$, and lanthanide mass fractions of $\approx 0.1$ the ejecta reach optically thin conditions at times between $0.7-1.5 \mathrm{~d}$, while during that epoch they produce bolometric luminosites of $\sim$ $3-7 \times 10^{40} \mathrm{erg} \mathrm{s}^{-1}$, which peak in near-IR frequency bands, and they exhibit photospheric temperatures within $2.2-2.8 \times 10^{3} \mathrm{~K}$. Since the composition is rather similar for the four regular models, the observed differences of peak times and luminosities between these models are a result mainly of the different ejecta masses. A softer EoS, as well as a more asymmetric binary produce slightly more luminous transients with longer durations.

(iii) The fluxes received by observers increase by a factor of 1.5-3 when going from equatorial to polar viewing angles, while for different configurations this factor increases with the level of oblateness of the ejecta. The spectra differ only marginally for different observer angles.

(iv) Neglecting all neutrino interactions after both NSs first touched for a symmetric model with DD2 EoS reduces the average electron fraction from 0.27 to 0.13 , increases the lanthanide fractions by a factor of about 2 , and moves radioactive heating power effectively from times $t<1 \mathrm{~d}$ to $t>1 \mathrm{~d}$. The main consequences for the kilonova are an extended peak (by $\sim 15$ per cent) and a luminosity reduction (by $\sim 40$ per cent), while the spectrum is only mildly redshifted. These changes are comparable to the changes obtained when reducing $Y_{e}$ by 0.1 globally for all trajectories. Conversely, increasing $Y_{e}$ by 0.1 reduces the lanthanide fractions by a factor of 4 , but at the same time reduces the heating rate at all times by about $20-40$ per cent. Hence, the emission is only marginally more luminous, but its duration is $\sim 20$ per cent shorter and the spectrum shifted towards the blue end of the near-IR band. The fact that the impact of these $Y_{e}$ modifications is of comparable magnitude as the variations due to different EoSs or binary mass ratios implies that neutrino interactions must be properly accounted for in models that shall be used to reliably infer the ejecta properties.

(v) None of our investigated models produces a transient compatible with the blue or red component of AT2017gfo that accompanied GW170817. Agreement with the high spectral temperatures and high luminosities of the early, blue component of AT2017gfo would require significantly smaller lanthanide mass fractions, and therefore opacities, than found for our models. While the low spectral temperatures of our models are roughly compatible with the late, red component of AT2017gfo, the short duration and low luminosity of our kilonova signals would call for a significantly higher ejecta mass 
than the $0.009 \mathrm{M}_{\odot}$ of our most massive case. However, our results are not conclusive regarding the role of dynamical ejecta in AT2017gfo as they cover only a small set of EoSs and binary parameters allowed by GW170817 and do not include secular post-merger ejecta.

(vi) We found that one-zone kilonova models, which assume that the nucleosynthesis results for a single trajectory are representative for the bulk of the ejecta, tend to systematically underestimate the lanthanide fractions, and therefore the opacities, compared to models adopting an ensemble of trajectories that samples a range of thermodynamic conditions. Moreover, the heating rates predicted by one-zone models may depend rather sensitively on the particular choice of thermodynamic conditions along the trajectory and on the employed nuclear-physics input, in contrast to which the average heating rate for an ensemble of trajectories was found to be more robust with respect to the variation of global parameters.

The fact that our kilonova models based on single-component dynamical-ejecta configurations are unable to reproduce the blue or the red component of AT2017gfo is in line with previous analyses, such as Siegel (2019); Nedora et al. (2021). We note, however, that these works are based on a comparison with the Arnett-type (i.e. essentially spherically symmetric one-zone) model of AT2017gfo by Villar et al. (2017). Since radiative transfer models can predict quite different peak properties for specific observer directions than one-zone models - particularly in the case of multicomponent ejecta (Korobkin et al. 2021) - the masses, bulk velocities, and bulk opacities found in Villar et al. (2017) for the different components of AT2017gfo should be taken with care.

The role of the dynamical ejecta in AT2017gfo may not be constrained to the minor effects suggested by the single-component models considered in our study. First, our set of models considers only very few EoSs and binary mass ratios, while more extreme configurations may lead to significantly more massive and/or lanthanidepoor ejecta and correspondingly brighter and blue-shifted kilonova signals. Another, more general argument may be connected to the tendency ${ }^{16}$ that dynamical ejecta are expelled in all directions, albeit with a greater mass loading in equatorial than polar directions (Bauswein et al. 2013; Sekiguchi et al. 2015; Radice et al. 2018b; Kullmann et al. 2021). The dynamical ejecta should therefore overcast all non-relativistic post-merger ejecta to a substantial extent, such that a reprocessing of the emission from interior ejecta by the enveloping dynamical ejecta can be naturally expected, in particular at early times $(t \lesssim 1-1.5 \mathrm{~d})$ when most of the dynamical ejecta are still optically thick. The model by Kawaguchi et al. (2018), which is based on an analytic distribution of multicomponent ejecta, supports this notion, and it demonstrated that the blue component of AT2017gfo can be produced even by a lanthanide-rich dynamicalejecta component, if the latter is subject to heating by photons coming from an enshrouded cloud of lanthanide-poor post-merger ejecta. Even though this model did not adopt a self-consistent ejecta configuration from a hydrodynamic simulation, the results highlight the difficulty of connecting specific kilonova (e.g. blue or red)

\footnotetext{
${ }^{16}$ We note that kilonova studies (e.g. Wollaeger et al. 2018) informed by Newtonian merger models (e.g. Rosswog et al. 2014) often consider purely toroidal geometries to represent the dynamical ejecta. This is because matter ejection into polar directions as a result of collision shocks and corresponding heating is typically less pronounced in Newtonian simulations compared to the general relativistic case, mainly because of effectively weaker effects by the gravitational interaction of the colliding bodies.
}

components to individual (e.g. dynamical or viscous or neutrinodriven) ejecta components, because a kilonova from multiple ejecta components may not just be the sum of kilonovae from singlecomponent ejecta models.

Keeping the aforementioned aspects in mind, we also mention some alternative scenarios that could explain the blue component of AT2017gfo. The partial disruption of the dynamical ejecta due to a relativistic jet and its corresponding energy injection have been considered as a possible way to make the ejecta shine brighter and at higher temperatures (Nativi et al. 2020; Klion et al. 2021b). Moreover, neutrino oscillations could change the nucleosynthesis signature of the ejecta and reduce their lanthanide content (e.g. Zhu, Perego \& McLaughlin 2016; George et al. 2020). Apart from the dynamical ejecta and subsequent neutrino-driven winds from the surface of the hyper-massive NS, additional fast and lanthanide-poor material could be ejected in connection to magnetic-field effects (e.g. Metzger, Thompson \& Quataert 2018; Mösta et al. 2020; Shibata, Fujibayashi \& Sekiguchi 2021) or in the form of a spiral-wave wind powered by an $m=1$ mode (Nedora et al. 2019). Future, more refined neutrino-magneto-hydrodynamic models of the merger and its remnant, as well as self-consistent kilonova calculations based on these models will have to reduce the large uncertainties that, as of yet, prohibit a conclusive assessment of the constituents that shaped the kilonova in GW170817.

Although the number of investigated models is small, our results provide a rough idea about the level of variation that is connected to different possible EoSs, binary mass ratios, and uncertainties in the treatment of neutrino transport. In our models the composition, and therefore the opacity and heating rate, depend only mildly on the EoS and mass ratio. Partially as a result of this circumstance, the analytic scaling formulae, equations (31), (33), and (34), describe relative differences of the peak properties between models with different ejecta masses, $M_{\mathrm{ej}}$, and different bulk velocities, $\langle v\rangle$, reasonably well. Hence, the dependence of the kilonova peak on global parameters of the merger can, to first order, be obtained by feeding the results for $M_{\mathrm{ej}}$ and $\langle v\rangle$ known from detailed merger simulations (e.g. Bauswein et al. 2013; Radice et al. 2018b; Krüger \& Foucart 2020; Nedora et al. 2020) into equations (31), (33), and (34). In a similar manner, the analytic formulae can also be used to approximately translate yet existing uncertainties from neutrino-hydrodynamic and nucleosynthesis modelling into error bars for the kilonova signal. We refer to section 5 of Part I for a discussion of uncertainties and detailed comparison with literature results for the ejecta properties.

Finally, we summarize the shortcomings of our study that should be improved upon in future work: Since the M1 approximation is less reliable in the optically thin regime, our models do not have the same predictive power as kilonova calculations based on Boltzmann solvers, particularly concerning the emission into specific directions. Also, we only include the early, dynamical ejecta here, but additional ejecta components produced by the merger remnant should be included as well, ideally accounting for the dynamical and radiative interactions between components. Moreover, the transition between the phase of matter ejection and homologous expansion is not included but should be accounted for to ensure a correct mass distribution as input for the kilonova calculation. Finally, our phenomenological treatment of the opacities is not based on atomic properties, which themselves depend on the local thermodynamic conditions. Our scheme could readily be improved by using an opacity framework based on atomic-physics models. 


\section{ACKNOWLEDGEMENTS}

$\mathrm{OJ}, \mathrm{AB}$, and $\mathrm{CC}$ acknowledge support by the European Research Council (ERC) under the European Union's Horizon 2020 research and innovation programme under grant agreement no. 759253 and by the - Project-ID 279384907 - Sonderforschungsbereich SFB 1245 by the Deutsche Forschungsgemeinschaft (DFG; German Research Foundation). OJ was partially supported by JSPS Grantsin-Aid for Scientific Research KAKENHI (A) $19 H 00693$ and by the Interdisciplinary Theoretical and Mathematical Sciences Program (iTHEMS) of RIKEN. SG acknowledges financial support from F.R.S. - FNRS (Belgium). This work has been supported by the Fonds de la Recherche Scientifique (FNRS; Belgium) and the Research Foundation Flanders (FWO; Belgium) under the EoS Project nr O022818F. AB was additionally supported by - ProjectID 138713538 - SFB 881 ('The Milky Way System', subproject A10) and by the State of Hesse within the Cluster Project ELEMENTS. At Garching, funding by the European Research Council through Grant ERC-AdG no. 341157-COCO2CASA and by the DFG through SFB-1258 'Neutrinos and Dark Matter in Astro- and Particle Physics (NDM)' and under Germany's Excellence Strategy through Cluster of Excellence ORIGINS (EXC-2094) - 390783311 is acknowledged. OJ acknowledges computational support by the HOKUSAI supercomputer at RIKEN/Japan and by the Max-Planck Computing and Data Facility (MPCDF) at Garching/Germany. The nucleosynthesis calculations benefited from computational resources made available on the Tier-1 supercomputer of the Fédération Wallonie-Bruxelles, infrastructure funded by the Walloon Region under the grant agreement no. 1117545 and the Consortium des Équipements de Calcul Intensif (CÉCI), funded by the (F.R.S. FNRS) under grant no. 2.5020.11 and by the Walloon Region.

\section{DATA AVAILABILITY}

The data underlying this article will be shared on reasonable request to the corresponding author.

\section{REFERENCES}

Abbott B. P. et al., 2017, Phys. Rev. Lett., 119, 161101

Ardevol-Pulpillo R., Janka H.-T., Just O., Bauswein A., 2019, MNRAS, 485, 4754

Arnett W. D., 1982, ApJ, 253, 785

Arnould M., Goriely S., 2020, Prog. Part. Nucl. Phys., 112, 103766

Audit E., Charrier P., Chièze J., Dubroca B., 2002, preprint (arXiv:astro-ph /0206281)

Banerjee S., Tanaka M., Kawaguchi K., Kato D., Gaigalas G., 2020, ApJ, 901, 29

Barbieri C., Salafia O. S., Perego A., Colpi M., Ghirlanda G., 2019, A\&A, 625, A152

Barnes J., Kasen D., Wu M.-R., Martínez-Pinedo G., 2016, ApJ, 829, 110

Barnes J., Zhu Y. L., Lund K. A., Sprouse T. M., Vassh N., McLaughlin G. C., Mumpower M. R., Surman R., 2021, ApJ, 918, 44

Bauswein A., Goriely S., Janka H.-T., 2013, ApJ, 773, 78

Bauswein A., Just O., Janka H.-T., Stergioulas N., 2017, ApJ, 850, L34

Breschi M., Perego A., Bernuzzi S., Del Pozzo W., Nedora V., Radice D., Vescovi D., 2021, MNRAS, 505, 1661

Bulla M., 2019, MNRAS, 489, 5037

Cernohorsky J., Bludman S. A., 1994, ApJ, 433, 250

Chornock R. et al., 2017, ApJ, 848, L19

Ciolfi R., Kalinani J. V., 2020, ApJ, 900, L35

Côté B. et al., 2018, ApJ, 855, 99

Coughlin M. W. et al., 2018, MNRAS, 480, 3871
Cowan J. J., Sneden C., Lawler J. E., Aprahamian A., Wiescher M., Langanke K., Martínez-Pinedo G., Thielemann F.-K., 2021, Rev. Mod. Phys., 93, 015002

Darbha S., Kasen D., 2020, ApJ, 897, 150

Eichler D., Livio M., Piran T., Schramm D. N., 1989, Nature, 340, 126

Even W. et al., 2020, ApJ, 899, 24

Fernández R., Metzger B. D., 2013, MNRAS, 435, 502

Fernández R., Quataert E., Schwab J., Kasen D., Rosswog S., 2015, MNRAS, 449, 390

Fontes C. J., Fryer C. L., Hungerford A. L., Wollaeger R. T., Korobkin O., 2020, MNRAS, 493, 4143

Foucart F., O'Connor E., Roberts L., Kidder L. E., Pfeiffer H. P., Scheel M. A., 2016, Phys. Rev. D, 94, 123016

Foucart F., Duez M. D., Kidder L. E., Nguyen R., Pfeiffer H. P., Scheel M. A., 2018, Phys. Rev. D, 98, 063007

Foucart F., Duez M. D., Hebert F., Kidder L. E., Pfeiffer H. P., Scheel M. A., 2020, ApJ, 902, L27

Frebel A., Beers T. C., 2018, Phys. Today, 71, 30

Freiburghaus C., Rosswog S., Thielemann F.-K., 1999, ApJ, 525, L121

Fujibayashi S., Kiuchi K., Nishimura N., Sekiguchi Y., Shibata M., 2018, ApJ, 860, 64

García-Senz D., Relaño A., Cabezón R. M., Bravo E., 2009, MNRAS, 392, 346

George M., Wu M.-R., Tamborra I., Ardevol-Pulpillo R., Janka H.-T., 2020, Phys. Rev. D, 102, 103015

Goriely S., 2015, Eur. Phys. J. A, 51, 22

Goriely S., Bauswein A., Janka H.-T., 2011, ApJ, 738, L32 +

Goriely S., Sida J.-L., Lemaître J.-F., Panebianco S., Dubray N., Hilaire S., Bauswein A., Janka H.-T., 2013, Phys. Rev. Lett., 111, 242502

Goriely S., Bauswein A., Just O., Pllumbi E., Janka H.-T., 2015, MNRAS, 452, 3894

Gottlieb O., Nakar E., 2021, preprint (arXiv:2106.03860)

Grossman D., Korobkin O., Rosswog S., Piran T., 2014, MNRAS, 439, 757

Hamidani H., Kiuchi K., Ioka K., 2020, MNRAS, 491, 3192

Heinzel J. et al., 2021, MNRAS, 502, 3057

Hoffman R. D., Woosley S. E., Qian Y.-Z., 1997, ApJ, 482, 951

Hotokezaka K., Nakar E., 2020, ApJ, 891, 152

Hotokezaka K., Kiuchi K., Kyutoku K., Okawa H., Sekiguchi Y.-i., Shibata M., Taniguchi K., 2013, Phys. Rev. D, 87, 024001

Ishizaki W., Kiuchi K., Ioka K., Wanajo S., 2021, ApJ, 922, 185

Ito H., Just O., Takei Y., Nagataki S., 2021, ApJ, 918, 59

Just O., Bauswein A., Pulpillo R. A., Goriely S., Janka H.-T., 2015a, MNRAS, 448,541

Just O., Obergaulinger M., Janka H.-T., 2015b, MNRAS, 453, 3386

Just O., Obergaulinger M., Janka H.-T., Bauswein A., Schwarz N., 2016, ApJ, 816 , L30

Just O., Goriely S., Janka H.-T., Nagataki S., Bauswein A., 2022, MNRAS, 509, 1377

Kajino T., Aoki W., Balantekin A. B., Diehl R., Famiano M. A., Mathews G. J., 2019, Prog. Part. Nucl. Phys., 107, 109

Kasen D., Barnes J., 2019, ApJ, 876, 128

Kasen D., Thomas R. C., Nugent P., 2006, ApJ, 651, 366

Kasen D., Badnell N. R., Barnes J., 2013, ApJ, 774, 25

Kasen D., Fernández R., Metzger B. D., 2015, MNRAS, 450, 1777

Kasen D., Metzger B., Barnes J., Quataert E., Ramirez-Ruiz E., 2017, Nature, 551,80

Kawaguchi K., Shibata M., Tanaka M., 2018, ApJ, 865, L21

Kawaguchi K., Shibata M., Tanaka M., 2020, ApJ, 889, 171

Kawaguchi K., Fujibayashi S., Shibata M., Tanaka M., Wanajo S., 2021, ApJ, 913, 100

Klion H., Tchekhovskoy A., Kasen D., Kathirgamaraju A., Quataert E., Fernández R., 2021a, preprint (arXiv:2108.04251)

Klion H., Duffell P. C., Kasen D., Quataert E., 2021b, MNRAS, 502, 865

Korobkin O., Rosswog S., Arcones A., Winteler C., 2012, MNRAS, 426, 1940

Korobkin O. et al., 2021, ApJ, 910, 116

Krüger C. J., Foucart F., 2020, Phys. Rev. D, 101, 103002

Kulkarni S. R., 2005, preprint (arXiv:astro-ph/0510256) 
Kullmann I., Goriely S., Just O., Ardevol-Pulpillo R., Bauswein A., Janka H. T., 2021, preprint (arXiv:2109.02509) (Part I)

Kuroda T., Takiwaki T., Kotake K., 2016, ApJS, 222, 20

Lattimer J. M., Schramm D. N., 1976, ApJ, 210, 549

Lemaître J. F., Goriely S., Bauswein A., Janka H. T., 2021, Phys. Rev. C, 103, 025806

Levermore C. D., 1984, J. Quant. Spec. Radiat. Transf., 31, 149

Li L.-X., Paczyński B., 1998, ApJ, 507, L59

Lippuner J., Roberts L. F., 2015, ApJ, 815, 82

Lippuner J., Roberts L. F., 2017, ApJS, 233, 18

Lippuner J., Fernández R., Roberts L. F., Foucart F., Kasen D., Metzger B. D., Ott C. D., 2017, MNRAS, 472, 904

Lucy L. B., 2005, A\&A, 429, 19

Margalit B., Metzger B. D., 2017, ApJ, 850, L19

Martin D., Perego A., Arcones A., Thielemann F.-K., Korobkin O., Rosswog S., 2015, ApJ, 813, 2

Martin D., Perego A., Kastaun W., Arcones A., 2018, Class. Quantum Gravity, 35,034001

McKinney J. C., Tchekhovskoy A., Sadowski A., Narayan R., 2014, MNRAS, 441,3177

Metzger B. D., 2019, Living Rev. Relativ., 23, 1

Metzger B. D., Fernández R., 2014, MNRAS, 441, 3444

Metzger B. D. et al., 2010, MNRAS, 406, 2650

Metzger B. D., Bauswein A., Goriely S., Kasen D., 2015, MNRAS, 446, 1115

Metzger B. D., Thompson T. A., Quataert E., 2018, ApJ, 856, 101

Mihalas D., Mihalas B. W., 1984, Foundations of Radiation Hydrodynamics. Oxford Univ. Press, New York

Miller J. M. et al., 2019, Phys. Rev. D, 100, 023008

Minerbo G. N., 1978, J. Quant. Spec. Radiat. Transf., 20, 541

Monaghan J. J., 1992, ARA\&A, 30, 543

Mösta P., Radice D., Haas R., Schnetter E., Bernuzzi S., 2020, ApJ, 901, L37

Mumpower M. R., Surman R., Fang D.-L., Beard M., Möller P., Kawano T., Aprahamian A., 2015, Phys. Rev. C, 92, 035807

Nativi L., Bulla M., Rosswog S., Lundman C., Kowal G., Gizzi D., Lamb G. P., Perego A., 2020, MNRAS, 500, 1772

Nedora V., Bernuzzi S., Radice D., Perego A., Endrizzi A., Ortiz N., 2019, ApJ, 886, L30

Nedora V. et al., 2020, preprint (arXiv:2011.11110)

Nedora V. et al., 2021, ApJ, 906, 98

Nicholl M. et al., 2017, ApJ, 848, L18

O'Connor E., 2015, ApJS, 219, 24

Palenzuela C., Liebling S. L., Neilsen D., Lehner L., Caballero O. L., O’Connor E., Anderson M., 2015, Phys. Rev. D, 92, 044045

Perego A., Rosswog S., Cabezón R. M., Korobkin O., Käppeli R., Arcones A., Liebendörfer M., 2014, MNRAS, 443, 3134

Perego A., Radice D., Bernuzzi S., 2017, ApJ, 850, L37

Pinto P. A., Eastman R. G., 2000, ApJ, 530, 744

Pons J. A., Ibáñez J. M., Miralles J. A., 2000, MNRAS, 317, 550

Price D. J., Monaghan J. J., 2007, MNRAS, 374, 1347

Radice D., Perego A., Zappa F., Bernuzzi S., 2018a, ApJ, 852, L29

Radice D., Perego A., Hotokezaka K., Fromm S. A., Bernuzzi S., Roberts L. F., 2018b, ApJ, 869, 130

Rezzolla L., Most E. R., Weih L. R., 2018, ApJ, 852, L25

Roberts L. F., Kasen D., Lee W. H., Ramirez-Ruiz E., 2011, ApJ, 736, L21 +

Rosswog S., Liebendörfer M., Thielemann F.-K., Davies M. B., Benz W., Piran T., 1999, A\&A, 341, 499

Rosswog S., Korobkin O., Arcones A., Thielemann F.-K., Piran T., 2014, MNRAS, 439, 744

Rosswog S., Feindt U., Korobkin O., Wu M.-R., Sollerman J., Goobar A., Martinez-Pinedo G., 2017, Class. Quantum Gravity, 34, 104001

Rosswog S., Sollerman J., Feindt U., Goobar A., Korobkin O., Wollaeger R., Fremling C., Kasliwal M. M., 2018, A\&A, 615, A132

Ruffert M., Janka H.-T., Schaefer G., 1996, A\&A, 311, 532

Ruffert M., Janka H.-T., Takahashi K., Schaefer G., 1997, A\&A, 319, 122
Sekiguchi Y., Kiuchi K., Kyutoku K., Shibata M., 2015, Phys. Rev. D, 91, 064059

Sekiguchi Y., Kiuchi K., Kyutoku K., Shibata M., Taniguchi K., 2016, Phys. Rev. D, 93, 124046

Shen S., Cooke R. J., Ramirez-Ruiz E., Madau P., Mayer L., Guedes J., 2015, ApJ, 807, 115

Shibata M., Zhou E., Kiuchi K., Fujibayashi S., 2019, Phys. Rev. D, 100, 023015

Shibata M., Fujibayashi S., Sekiguchi Y., 2021, Phys. Rev. D, 104, 063026

Siegel D. M., 2019, Eur. Phys. J. A, 55, 203

Siegel D. M., Metzger B. D., 2017, Phys. Rev. Lett., 119, 231102

Smartt S. J. et al., 2017, Nature, 551, 75

Smit J. M., van den Horn L. J., Bludman S. A., 2000, A\&A, 356, 559

Steiner A. W., Hempel M., Fischer T., 2013, ApJ, 774, 17

Stoitsov M. V., Dobaczewski J., Nazarewicz W., Pittel S., Dean D. J., 2003, Phys. Rev. C, 68, 054312

Sumiyoshi K., Fujibayashi S., Sekiguchi Y., Shibata M., 2021, ApJ, 907, 92

Tanaka M., Hotokezaka K., 2013, ApJ, 775, 113

Tanaka M. et al., 2017, PASJ, 69, 102

Tanaka M., Kato D., Gaigalas G., Kawaguchi K., 2020, MNRAS, 496, 1369

Tanvir N. R. et al., 2017, ApJ, 848, L27

Typel S., Röpke G., Klähn T., Blaschke D., Wolter H. H., 2010, Phys. Rev. C, 81, 015803

van de Voort F., Pakmor R., Grand R. J. J., Springel V., Gómez F. A., Marinacci F., 2020, MNRAS, 494, 4867

Vangioni E., Goriely S., Daigne F., François P., Belczynski K., 2016, MNRAS, 455,17

Villar V. A. et al., 2017, ApJ, 851, L21

Wanajo S., 2018, ApJ, 868, 65

Wanajo S., Sekiguchi Y., Nishimura N., Kiuchi K., Kyutoku K., Shibata M., 2014, ApJ, 789, L39

Watson D. et al., 2019, Nature, 574, 497

Waxman E., Ofek E. O., Kushnir D., Gal-Yam A., 2018, MNRAS, 481, 3423

Waxman E., Ofek E. O., Kushnir D., 2019, ApJ, 878, 93

Weih L. R., Gabbana A., Simeoni D., Rezzolla L., Succi S., Tripiccione R., 2020, MNRAS, 498, 3374

Wollaeger R. T. et al., 2018, MNRAS, 478, 3298

Wollaeger R. T. et al., 2021, ApJ, 918, 10

Wu M.-R., Fernández R., Martínez-Pinedo G., Metzger B. D., 2016, MNRAS, 463,2323

Wu M.-R., Barnes J., Martínez-Pinedo G., Metzger B. D., 2019, Phys. Rev. Lett., 122, 062701

Zhu Y. L., Perego A., McLaughlin G. C., 2016, Phys. Rev. D, 94, 105006

Zhu Y. L., Lund K. A., Barnes J., Sprouse T. M., Vassh N., McLaughlin G. C., Mumpower M. R., Surman R., 2021, ApJ, 906, 94

\section{APPENDIX A: OPACITY CALIBRATION}

Our kilonova treatment avoids the complexity connected to a microphysically consistent opacity, $\kappa$, which would need to account for thousands of transition lines between energy levels - most of them poorly known so far - of many isotopes in dependence of the detailed composition, density, temperature, and degree of ionization. Instead, we employ a simplified, grey version that parametrizes the opacity in terms of the lanthanide mass fraction, $X_{\mathrm{LA}}$, and temperature, $T$; see equations (13)-(15). The form of $\kappa$ was calibrated ${ }^{17}$ in a way to reproduce, as accurately as possible, a set of bolometric light curves

\footnotetext{
${ }^{17}$ In order to prevent confusion, we note that a similar calibration was already presented in the appendix of Just et al. (2022), which suggested a slightly different form of $\kappa\left(X_{\mathrm{LA}}, T\right)$. The reason is that Just et al. (2022) did not correct their luminosities for frame-dependent effects as we do here (cf. Section 2.6) and, hence, needed different opacities in order to reproduce the same reference results. We recommend using the newer version presented here.
} 

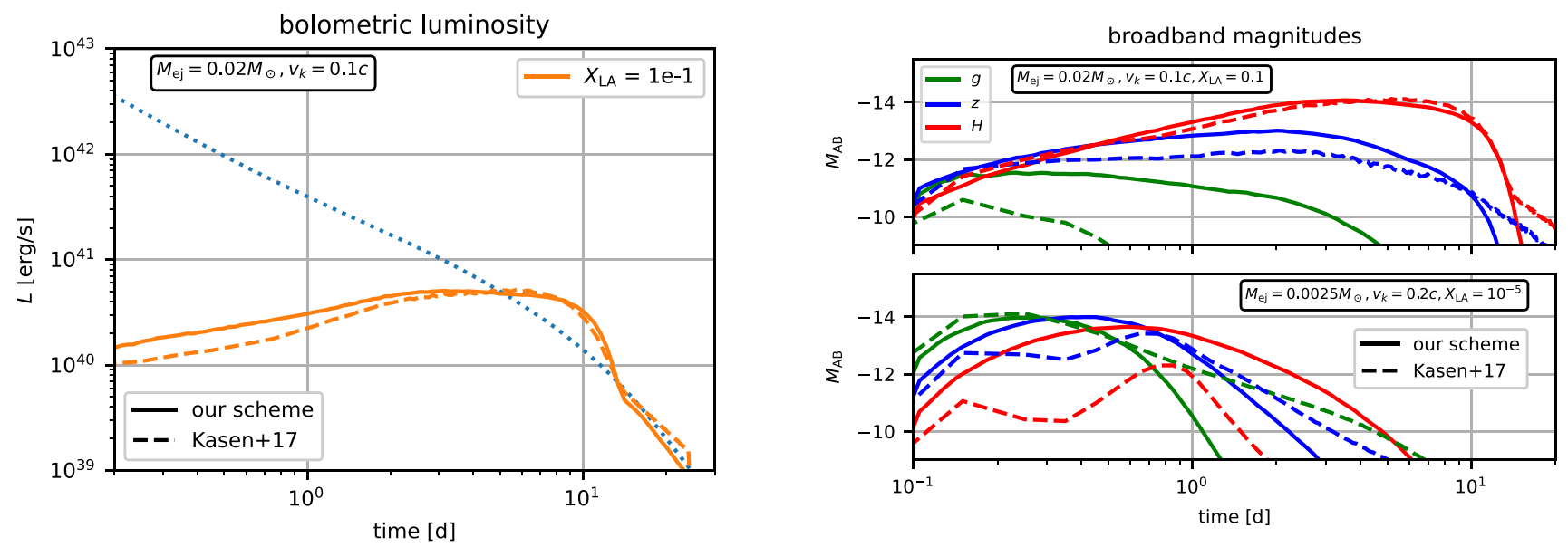

Figure A1. Left-hand panel: results of our opacity calibration using a set of bolometric light curves from Kasen et al. (2017). Solid lines refer to results obtained with our scheme, while dashed lines are the reference results from Kasen et al. (2017). Model parameters are listed in the insets. The blue dotted line denotes the effective heating rate after thermalization. Right-hand panel: comparison of broadband magnitudes in three exemplary frequency bands for two qualitatively different models. The top panel shows results for a model leading to a near-IR kilonova, while the model in the bottom panel produces a kilonova peaking rather in the optical regime. Despite quantitative differences, our scheme reproduces the peaks in the correct bands.

by Kasen et al. (2017) using a state-of-the-art opacity treatment, which differ by the contained mass of lanthanides. In Fig. A1, lefthand panel, we provide the light curves based on which we calibrated. The ejecta mass and bulk velocity for this reference case are $0.02 \mathrm{M}_{\odot}$ and $0.1 c$, respectively, while we refer to Kasen et al. (2017) for the precise form of the analytic mass distribution. For this test we use a radioactive heating rate that is presumably similar, though possibly not exactly identical, to the one used in Kasen et al. (2017). It is taken from Lippuner \& Roberts (2015) and given by $q_{\mathrm{rad}}\left[\mathrm{erg} \mathrm{g}^{-1} \mathrm{~s}^{-1}\right]=$ $\left(1.0763 \times 10^{10} t_{\text {day }}^{-1.518}+9.5483 \times 10^{9} e^{-t_{\text {day }} / 4.947}\right)$ with $t_{\text {day }}$ being the time in units of days. The homogeneous thermalization efficiency is computed, as in Kasen et al. (2017), using the analytic fit formula as function of the ejecta mass and velocity provided by Barnes et al. (2016).

A comparison of the peak behaviour of broadband light curves provides another consistency check for our treatment. In Fig. A1, we compare the broadband light curves in the $g, z$, and $H$ bands for two models. The model in the top panel of Fig. A1 assumes rather massive, relatively slow, and lanthanide-rich ejecta, and the peak is in the near-IR $(H)$ band. The model in the bottom panel assumes less massive ejecta with higher velocities and a low lanthanide fraction, and the peak is therefore at higher spectral temperatures, namely in the $g$ and $z$ bands. Albeit quantitative differences are noticeable, our scheme can reproduce the basic peak behaviour quite well. This result lends credibility to the broadband behaviour obtained in the dynamical ejecta models investigated in this paper.

\section{APPENDIX B: SENSITIVITY TO INITIAL CONDITIONS}

As argued in Section 2.3 , the light curve at $t \gtrsim 0.1 \mathrm{~d}$ is fairly independent of the choice of the initial conditions - namely the initialization time, $t_{0}$, as well as the initial distributions of radiation energy and gas temperature - as long as $t_{0}$ is chosen sufficiently early for the system to relax well before $t \sim 0.1 \mathrm{~d}$ into the correct state of balance between radioactive heating and adiabatic expansion. In this appendix, we back this argument by showing in Fig. B1 light curves for variations of the fiducial model, DD2-135135, using different initial conditions. All models in the main part of this study are initialized at $t_{0}=100 \mathrm{~s}$ with a constant temperature of $T_{0}=100 \mathrm{~K}$. The excellent agreement of the fiducial model with

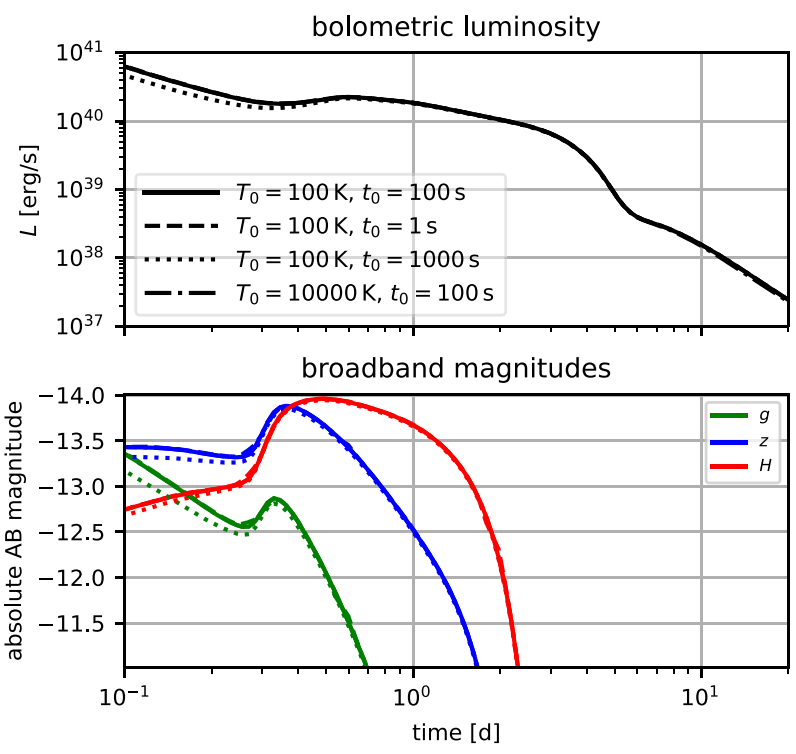

Figure B1. Comparison of bolometric luminosities (top panel) and broadband magnitudes (bottom panel) for kilonova calculations of model DD2135135 initialized with different gas temperatures, $T_{0}$, and at different times, $t_{0}$. Solid lines correspond to the standard case used for all models in the main part of this study. The lines corresponding to $t_{0}=100 \mathrm{~s}$ and $T_{0}=10000 \mathrm{~K}$ lie on top of the solid lines. Small differences at early times are only visible for the model initialized at $t_{0}=1000 \mathrm{~s}$.

This paper has been typeset from a $\mathrm{T}_{\mathrm{E}} \mathrm{X} / \mathrm{L} \mathrm{T} \mathrm{E} \mathrm{X}$ file prepared by the author. 
one model using instead $t_{0}=1 \mathrm{~s}$ and with another model using $T_{0}=10000 \mathrm{~K}$ supports the robustness of our results with respect to the initial conditions. Fig. B1 also reveals that a later initialization time of $t_{0}=1000 \mathrm{~s}$ would have led to a slight underestimation of the luminosities, though only at early times $t \lesssim 0.5 \mathrm{~d}$. 